\title{
Magnetic correlations and quantum criticality in the insulating antiferromagnetic, insulating spin liquid, renormalized Fermi liquid, and metallic antiferromagnetic phases of the Mott system $\mathrm{V}_{2} \mathrm{O}_{3}$
}

\author{
Wei Bao* \\ Physics Department, Brookhaven National Laboratory, Upton, New York 11973
}

C. Broholm

Department of Physics and Astronomy, The Johns Hopkins University, Baltimore, Maryland 21218

and Center for Neutron Research, National Institute of Standards and Technology, Gaithersburg, Maryland 20899

G. Aeppli

NEC, 4 Independence Way, Princeton, New Jersey 08540

S. A. Carter

Department of Physics, University of California, Santa Cruz, California 95064

P. Dai

Solid State Division, Oak Ridge National Laboratory, Oak Ridge, Tennessee 37831

T. F. Rosenbaum

James Franck Institute and Department of Physics, University of Chicago, Chicago, Illinois 60637

J. M. Honig and P. Metcalf

Department of Chemistry, Purdue University, West Lafayette, Indiana 47907

S. F. Trevino

United States Army Research Laboratory, Adelphi, Maryland 20783

and Center for Neutron Research, National Institute of Standards and Technology, Gaithersburg, Maryland 20899

(Received 28 April 1998)

\begin{abstract}
Magnetic correlations in all four phases of pure and doped vanadium sesquioxide $\left(\mathrm{V}_{2} \mathrm{O}_{3}\right)$ have been examined by magnetic thermal-neutron scattering. Specifically, we have studied the antiferromagnetic and paramagnetic phases of metallic $\mathrm{V}_{2-y} \mathrm{O}_{3}$, the antiferromagnetic insulating and paramagnetic metallic phases of stoichiometric $\mathrm{V}_{2} \mathrm{O}_{3}$, and the antiferromagnetic and paramagnetic phases of insulating $\mathrm{V}_{1.944} \mathrm{Cr}_{0.056} \mathrm{O}_{3}$. While the antiferromagnetic insulator can be accounted for by a localized Heisenberg spin model, the long-range order in the antiferromagnetic metal is an incommensurate spin-density wave, resulting from a Fermi surface nesting instability. Spin dynamics in the strongly correlated metal are dominated by spin fluctuations with a "single lobe" spectrum in the Stoner electron-hole continuum. Furthermore, our results in metallic $\mathrm{V}_{2} \mathrm{O}_{3}$ represent an unprecedentedly complete characterization of the spin fluctuations near a metallic quantum critical point, and provide quantitative support for the self-consistent renormalization theory for itinerant antiferromagnets in the small moment limit. Dynamic magnetic correlations for $\hbar \omega<k_{B} T$ in the paramagnetic insulator carry substantial magnetic spectral weight. However, they are extremely short-ranged, extending only to the nearest neighbors. The phase transition to the antiferromagnetic insulator, from the paramagnetic metal and the paramagnetic insulator, introduces a sudden switching of magnetic correlations to a different spatial periodicity which indicates a sudden change in the underlying spin Hamiltonian. To describe this phase transition and also the unusual short-range order in the paramagnetic state, it seems necessary to take into account the orbital degrees of freedom associated with the degenerate $d$ orbitals at the Fermi level in $\mathrm{V}_{2} \mathrm{O}_{3}$. [S0163-1829(98)06443-1]
\end{abstract}

\section{INTRODUCTION}

The Mott metal-insulator transition ${ }^{1}$ is a localization phenomenon driven by Coulomb repulsion between electrons. Mott insulators are common among transition-metal oxides and are an important group of materials which cannot be accounted for by conventional band theories of solids. Using a simplified model which now bears his name, ${ }^{2}$ Hubbard demonstrated a metal-insulator transition, in which a halffilled band is split by electronic correlations into upper (un- filled) and lower (filled) Hubbard bands. ${ }^{3}$ His approach, however, failed to produce a Fermi surface on the metallic side of the Mott transition. ${ }^{4}$ Also, there were unresolved questions concerning electronic spectral weight of the lower Hubbard band. ${ }^{5}$ Starting from the metallic side, a Fermi liquid description of the correlated metal was provided by Brinkman and Rice. ${ }^{6}$ They found that while the Pauli spin susceptibility, $\chi$, and the Sommerfeld constant, $\gamma$, are both strongly enhanced by Coulomb repulsion, the Wilson ratio, $\chi / \gamma$, remains constant. Such behavior reflects a strongly en- 
hanced effective mass and it was found that the metal to insulator transition could be associated with a divergence in the effective mass of these fermionic quasiparticles.

The discovery of high-temperature superconductivity in cuprates has revived interest in Mott systems. ${ }^{7}$ To be precise, the cuprates are classified as charge-transfer systems, ${ }^{8}$ but similar low-energy physics is expected for both classes of materials. Progress in experimental techniques, such as photoemission and $\mathrm{x}$-ray absorption spectroscopy, has recently enabled direct measurements of the Hubbard bands and the Brinkman-Rice resonance. ${ }^{9}$ On the theoretical side, there has also been progress especially in using infinite dimensional mean field theories ${ }^{10}$ to calculate the local spectral function of the Hubbard model close to the metal-insulator transition. ${ }^{11}$ A particularly important result is the synthesis of the seemingly contradictory pictures of Hubbard, BrinkmanRice, and Slater on the metal-insulator transition in strongly correlated electron systems. ${ }^{12}$ It is encouraging that these $D$ $=\infty$ theories to a large extent reproduce the experimentally observed features $^{9}$ in the electronic density of states for three-dimensional (3D) transition-metal oxides.

While there has been a remarkable convergence of experiments and theories which probe the electronic density of states across the Mott metal-insulator transition, little work has been done to explore magnetism close to this phase boundary for materials apart from the laminar cuprates. On the insulating side, the Heisenberg spin Hamiltonian was expected to offer a good description of magnetism, but as we shall see in the following, this turns out not to be the case for systems with degenerate atomic orbitals. On the metallic side of the transition, one might have hoped that the local spin picture would survive because charge carriers in the Brinkman-Rice liquid are heavy and nearly localized. As we shall see, this is also inconsistent with experiments. It turns out that the mobile quasiparticles have profound effects on magnetism in the metal. Indeed, the magnetism is that which one would associate with a quantum critical state, whose parameters are consistent with, for example, Moriya's selfconsistent renormalization (SCR) theory of metallic spin fluctuations. $^{13-18}$

We obtained these surprising conclusions and others to be discussed below through a neutron scattering study of magnetic correlations in $\mathrm{V}_{2} \mathrm{O}_{3}$ and its doped derivatives which constitute a famous 3D Mott system. ${ }^{19,1}$ Even though magnetic order occurs at low $T$ in the metallic and the insulating phases of $\mathrm{V}_{2} \mathrm{O}_{3}$, the material is the only known transitionmetal oxide to display a paramagnetic metal (PM) to paramagnetic insulator (PI) transition (see Fig. 1). The PM to antiferromagnetic insulator (AFI) transition in pure $\mathrm{V}_{2} \mathrm{O}_{3}$ is a spectacular first-order phase transition in which the resistivity abruptly increases by eight orders of magnitude, ${ }^{20,21}$ the lattice structure changes from trigonal to monoclinic, ${ }^{22,23}$ and the staggered moment immediately reaches over $80 \%$ of the low-temperature ordered moment. ${ }^{24}$ The mechanism for the phase transition remains controversial though it is clear that electron correlations are important because band-structure calculations predict a metallic state for both the trigonal and monoclinic crystal structures. ${ }^{25,26}$ One explanation for the transition is due to Slater, ${ }^{27}$ who argued that the antiferromagnetic transition doubles the unit cell which in turn opens a gap at the Fermi energy. This mechanism, however, cannot

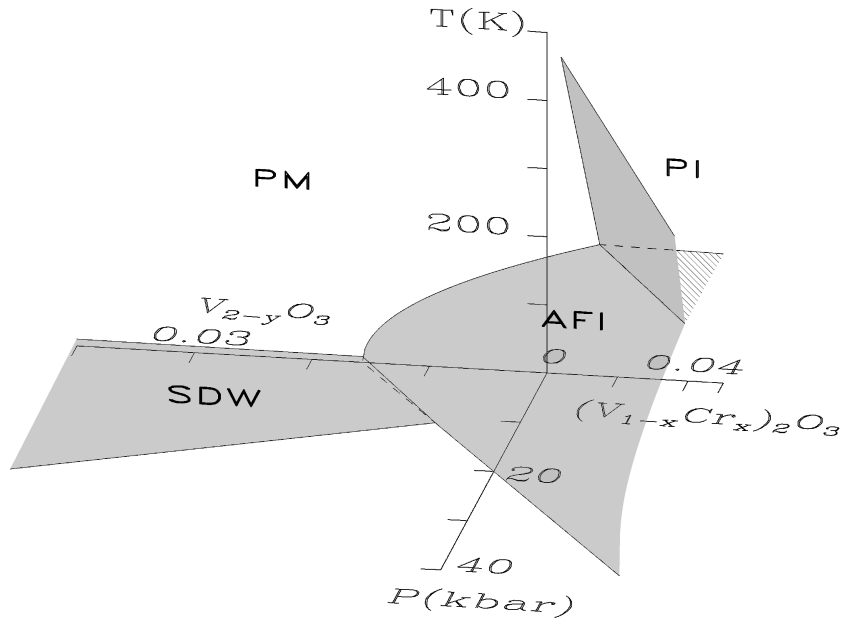

FIG. 1. The composition-pressure-temperature phase diagram of the $\mathrm{V}_{2} \mathrm{O}_{3}$ system (Refs. 19, 66, and 33). There exist four phases: the paramagnetic insulator (PI), the antiferromagnetic insulator (AFI), the paramagnetic metal (PM), and the spin-density-wave (SDW), also known as antiferromagnetic metal (AFM). The Mott transition between the PM and PI phases is first order, as well as the transitions to the AFI phase. The first-order nature of the latter phase transition becomes much less pronounced for $\mathrm{Cr}$ concentrations exceeding $x \sim 3 \%$. The transition to the SDW is second order. Except for the AFI phase, which is monoclinic, all other phases have the trigonal corundum structure.

account for the PM-PI transition and offers no explanation for the existence of the PI phase. Another viewpoint is that the AFI phase is simply the magnetically ordered state of the PI and that the Mott mechanism works for the PM-AFI transition as it does for the PM-PI transition. Following this approach, there have been various attempts to incorporate the antiferromagnetic phase in the framework of the Mott metalinsulator transition. ${ }^{28-30,12}$ However, none of the theories convincingly accounts for the first-order nature of the PI-AFI transition observed in $\mathrm{V}_{2} \mathrm{O}_{3}$. Another shortcoming of the theories is that they assume that low-energy and static magnetic correlations are characterized by the same wave vector throughout the phase diagram, something that our experiments show is not the case for the $\mathrm{V}_{2} \mathrm{O}_{3}$ system.

Magnetic order and spin waves below $25 \mathrm{meV}$ in the AFI phase of $\mathrm{V}_{2} \mathrm{O}_{3}$ were previously measured by other workers. $^{24,31,32}$ Our experiments are the first neutron scattering study of magnetic correlations in the AFM, PM, and PI phases of $\mathrm{V}_{2} \mathrm{O}_{3}$. Our most important conclusions are the following. (i) the AFM has a small moment incommensurate spin-density wave (SDW) at low temperatures, which results from a Fermi surface instability. (ii) Dynamic spin correlations in the entire PM phase are controlled by the same Fermi surface instability. (iii) Magnetic correlations in the metallic phases are those one might expect near a quantum critical point. They are quantitatively described by the SCR theory for itinerant antiferromagnetism in the small moment limit. (iv) Anomalously short-range dynamic spin correlations in the PI are closely related to those in the PM but are different from the magnetic order in the AFI. (v) Phase transitions to the AFI, from either the PM or the PI, cause an abrupt switch of the magnetic wave vector, signaling a sudden change of the exchange constants in the spin Hamil- 


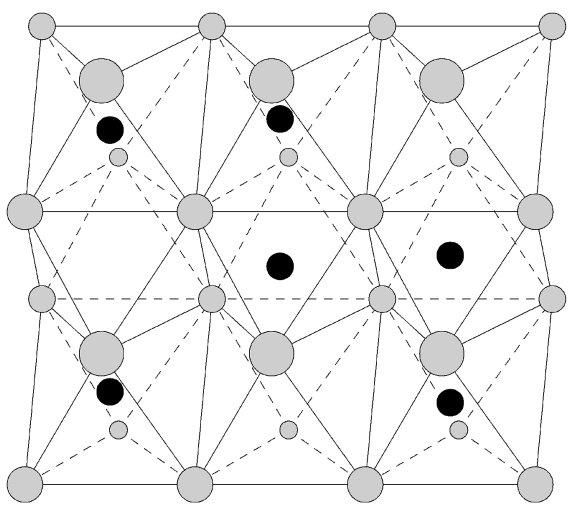

FIG. 2. The corundum lattice structure of $\mathrm{V}_{2} \mathrm{O}_{3}$. The solid symbols denote $\mathrm{V}$ ions and the hatched symbols denote $\mathrm{O}$ ions.

tonian. All of (i) $-(\mathrm{v})$ require more than a conventional Heisenberg spin Hamiltonian for a satisfactory explanation. In metallic samples the geometry of the Fermi surface directly affects magnetic correlations, while in insulating samples there are low-energy orbital degrees of freedom which are important for describing the unusual PI phase and the PI-AFI phase transition. Some of the above results were previously published in short reports. ${ }^{33-39}$

The organization of the paper is as follows. Section II covers experimental details concerning sample preparation and neutron scattering instrumentation, Secs. III, IV, V, and VI describe magnetic correlations in the AFM, PM, AFI, and PI phases, respectively, and Sec. VII concludes the paper with a discussion of the overall interpretation of the data.

\section{EXPERIMENTAL DETAILS}

$\mathrm{V}_{2} \mathrm{O}_{3}$ has the corundum structure ${ }^{40}$ (space group No. 165, $R \overline{3} c$, refer to Fig. 2) in all but the AFI phase, where small lattice distortions break the threefold rotation symmetry to yield a monoclinic structure. ${ }^{23} \mathrm{We}$ use the conventional hexagonal unit cell ${ }^{33,20}$ with six $\mathrm{V}_{2} \mathrm{O}_{3}$ formula units per unit cell to index real and reciprocal space. The reciprocal lattice parameters are $a^{*}=4 \pi / \sqrt{3} a=1.47(1) \AA^{-1}$ and $c^{*}=2 \pi / c$ $=0.448(1) \AA^{-1}$ in the metallic phases, $a^{*}=1.46(1) \AA^{-1}$ and $c^{*}=0.449(2) \AA^{-1}$ in the AFI phase, and $a^{*}$ $=1.45(1) \AA^{-1}$ and $c^{*}=0.451(2) \AA^{-1}$ in the PI phase. More accurate values of lattice parameters, as functions of temperature and doping, can be found in Refs. 22, 41 and 42. With this unit cell, the characteristic wave vector of the AFI spin structure ${ }^{24}$ is $(1 / 2,1 / 2,0)$, while it is $(0,0,1.7)$ for the SDW structure $^{33}$ (refer to Fig. 3). Samples were oriented in either the $(h h l)$ or the $(h 0 l)$ zone to reach peaks in the neutron scattering associated with the two types of magnetic correlations. Selection rules for nuclear Bragg points in these two zones are $(h h l): l=3 n$ and $(h 0 l): h-l=3 n, l=2 n$.

Single crystals of $\mathrm{V}_{2-y} \mathrm{O}_{3}$ were grown using a skull melter. ${ }^{43}$ The stoichiometry of pure $\mathrm{V}_{2} \mathrm{O}_{3}$ and metaldeficient $\mathrm{V}_{2-y} \mathrm{O}_{3}$ was controlled to within $\delta y=0.003$ by annealing sliced, as-grown $\mathrm{V}_{2} \mathrm{O}_{3}$ crystals in a suitably chosen $\mathrm{CO}-\mathrm{CO}_{2}$ atmosphere ${ }^{44}$ for two weeks at $1400^{\circ} \mathrm{C}$. Single crystals of $\left(\mathrm{V}_{1-x} \mathrm{Cr}_{x}\right)_{2} \mathrm{O}_{3}$ were grown using the Tri-arc technique. ${ }^{21}$ The compositions were determined by atomic absorption spectroscopy and wet chemical titration tech- (a) SDW

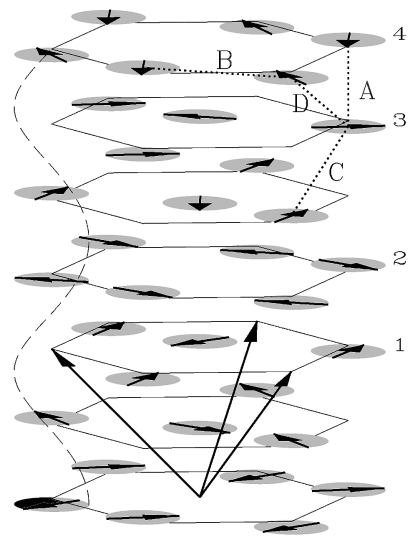

(b) AFI

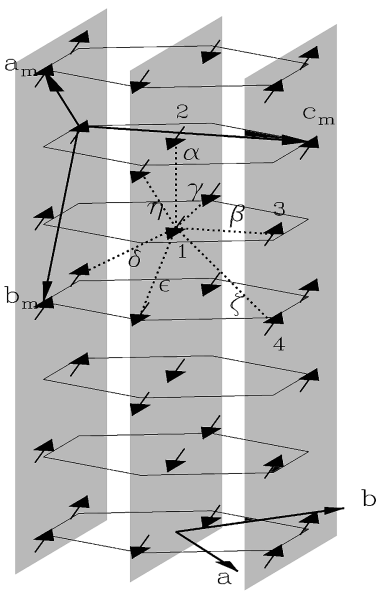

FIG. 3. (a) Spin structure in the SDW state (Ref. 33) of $\mathrm{V}_{2-y} \mathrm{O}_{3}$. Both spiral chiralities exist in our crystal. The staggered moment is $0.15 \mu_{B}$ per $\mathrm{V}$. The four types of near-neighbor spin pairs which are coupled by appreciable exchange interactions are indicated by $A-D$. (b) Spin structure in the AFI state (Ref. 24), which breaks the threefold symmetry of the corundum structure. The staggered moment is $1.2 \mu_{B}$ per V. $\alpha-\eta$ indicate seven distinct exchange constants. The conventional hexagonal unit cell is used in this paper. The primitive vectors $\mathbf{a}$ and $\mathbf{b}$ of this cell are shown in (b), and the c vector, which is not shown, spans the stack from bottom to top. The primitive vectors of the primitive trigonal unit cell are shown in (a), and the primitive vectors $\mathbf{a}_{m}, \mathbf{b}_{m}$, and $\mathbf{c}_{m}$ of the primitive monoclinic magnetic unit cell of the AFI are shown in (b). There are four spins (1-4) per primitive cell.

nique. The mass of samples for elastic neutron scattering was typically $100 \mathrm{mg}$, while we used several grams for inelastic neutron scattering experiments. Large single crystals were sliced into plates of $\sim 1.5 \mathrm{~mm}$ thickness as shown in Fig. 4(a) to allow oxygen diffusion to the bulk during the annealing process. Several single crystals of each composition were mutually aligned to increase the sensitivity for inelastic measurements. We report experiments for the seven compositions which are listed in Table I. The samples have been well characterized in previous studies using bulk measurement techniques. ${ }^{21,45-49}$

The sample temperature was controlled using liquid $\mathrm{He}$

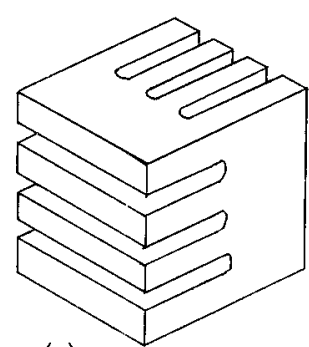

(a)

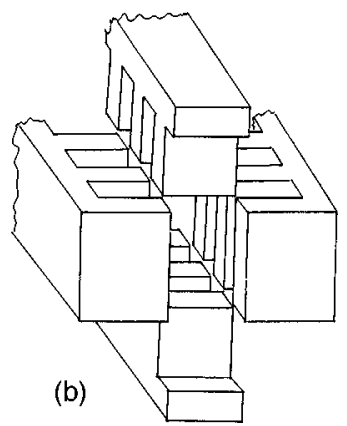

FIG. 4. (a) Cross-cut pattern used to facilitate oxygen diffusion in single crystals during annealing. (b) Adjustable neutron aperture, made of boron-polyethylene, to limit fast neutrons within small angles of the main beam. Two pairs of opposite "jaws" can move into each other to adjust the central opening according to the sample size. 
TABLE I. Samples used in this study. For easy location of the samples in the phase diagram (Fig. 1), $x$ or $y$ in molecular formula $\left(\mathrm{V}_{1-x} \mathrm{Cr}_{x}\right)_{2} \mathrm{O}_{3}$ or $\mathrm{V}_{2-y} \mathrm{O}_{3}$ is provided. The ambient transition temperature and physical states below and above the phase transition are also listed.

\begin{tabular}{lcccc}
\hline \hline & $y$ or $x$ & $T_{N}(\mathrm{~K})$ & $0 \mathrm{~K}$ & $300 \mathrm{~K}$ \\
\hline $\mathrm{V}_{1.963} \mathrm{O}_{3}$ & 0.037 & $8.4(1)$ & SDW & PM \\
$\mathrm{V}_{1.973} \mathrm{O}_{3}$ & 0.027 & $8.5(1)$ & SDW & PM \\
$\mathrm{V}_{1.983} \mathrm{O}_{3}$ & 0.017 & $9.5(1)$ & SDW & PM \\
$\mathrm{V}_{1.985} \mathrm{O}_{3}$ & 0.015 & $55(1)$ & AFI & PM \\
$\mathrm{V}_{1.988} \mathrm{O}_{3}$ & 0.012 & $70(2)$ & AFI & PM \\
$\mathrm{V}_{2} \mathrm{O}_{3}$ & 0.0 & $170(2)$ & AFI & PM \\
$\mathrm{V}_{1.944} \mathrm{Cr}_{0.056} \mathrm{O}_{3}$ & 0.028 & $180(2)$ & AFI & PI \\
\hline \hline
\end{tabular}

flow cryostats in the range from $1.4 \mathrm{~K}$ to $293 \mathrm{~K}$, or using displex closed-cycle cryostats from $10 \mathrm{~K}$ to $293 \mathrm{~K}$. With a heating element, the displex cryostat could reach temperatures up to $600 \mathrm{~K}$.

Hydrostatic pressure up to $8 \mathrm{kbar}$ was produced using a He gas high-pressure apparatus ${ }^{50}$ at NIST, which can be loaded into an "orange" ILL cryostat with a temperature range from $1.4 \mathrm{~K}$ to room temperature.

\section{A. Neutron scattering instrumentation}

Neutron scattering measurements were performed on triple-axis spectrometers BT2, BT4, and BT9 at the NIST research reactor, on $\mathrm{HB} 1$ at the High Flux Isotope Reactor (HFIR) of ORNL, and on H4M and H7 at the High Flux Beam Reactor (HFBR) of BNL. For unpolarized neutron experiments, we used a Be (101) monochromator on $\mathrm{HB} 1, \mathrm{Cu}$ (220) and pyrolytic graphite (PG) (002) monochromators on BT4, and PG (002) monochromators on all other instruments. PG (002) analyzers were used for all unpolarized triple-axis experiments. For polarized neutron measurements on BT2, crystals of Heusler alloy $\mathrm{Cu}_{2} \mathrm{MnAl}[\mathrm{d}(111)$ $=3.445 \AA$ ] were used as a monochromator and analyzer. Fast neutrons were removed from the incident beam by an in-pile sapphire filter ${ }^{51}$ on $\mathrm{HB} 1$, and PG filters ${ }^{52}$ were used to remove high-order neutron contaminations where appropriate. The horizontal collimations were controlled with Soller slits and are specified in the figures containing the experimental data. The fast neutron background at BT4 in the small scattering angle limit was reduced by a $30 \mathrm{~cm}$ long boronpolyethylene aperture before the sample and a similar $15 \mathrm{~cm}$ long aperture after the sample. The opening of the aperture was adjusted to match the sample size [Fig. 4(b)]. These apertures suppress fast neutron background in the small angle limit which is important for measuring high-energy inelastic magnetic scattering.

In most of our scans, the background is approximately constant. For some scans, however, the scattering angle falls below $\approx 7^{\circ}$ where background increases rapidly with decreasing angle. For example, a constant $\hbar \omega=28 \mathrm{meV}$ scan measured with $\mathrm{H} 4 \mathrm{M}$ is shown in Fig. 5 with solid circles. The scattering angle at (100) is $5.4^{\circ}$. As a result, the background (open symbols), measured with the analyzer turned $10^{\circ}$ from the reflection condition, increases near (100). The solid line represents data with this extra background contribution subtracted. ${ }^{53}$

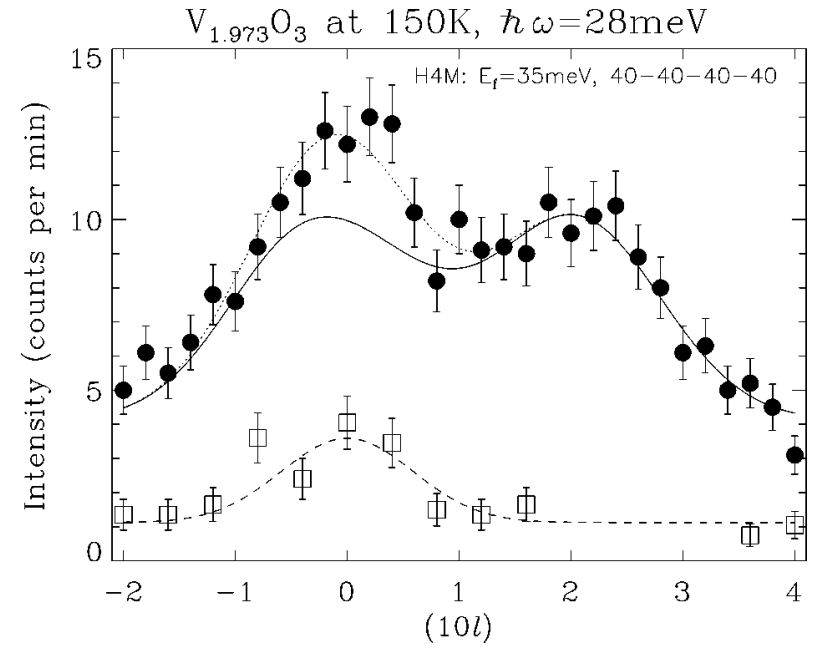

FIG. 5. A constant energy scan (solid circles) for which the scattering angle near (100) is close to the incident neutron beam. The squares denote the background measured with the analyzer rotated $10^{\circ}$ from the reflection condition. See text for details.

\section{B. Neutron scattering cross section}

The magnetic neutron scattering cross section for momentum transfer $\hbar \mathbf{q}=\hbar\left(\mathbf{k}-\mathbf{k}^{\prime}\right)$ and energy transfer $\hbar \omega$ is given by $^{54}$

$$
\begin{aligned}
\frac{d^{2} \sigma}{d \Omega d E^{\prime}}= & \frac{k^{\prime}}{k}\left(\frac{\gamma r_{0}}{2}\right)^{2}[f(\mathbf{q})]^{2} e^{-2 W(\mathbf{q})} \\
& \times \sum_{\alpha \beta}\left(\delta_{\alpha \beta}-\hat{q}_{\alpha} \hat{q}_{\beta}\right) N \mathcal{S}^{\alpha \beta}(\mathbf{q}, \omega),
\end{aligned}
$$

where $N$ is the number of hexagonal unit cells, $\left(\gamma r_{0} / 2\right)^{2}$ $=72.65 \times 10^{-3} \mathrm{barn} / \mu_{B}^{2}, f(\mathbf{q})$ is the magnetic form factor for the $\mathrm{V}^{3+}$ ion, ${ }^{55} \exp [-2 W(\mathbf{q})]$ is the Debye-Waller factor, and the dynamical structure factor is given by

$$
\begin{aligned}
\mathcal{S}^{\alpha \beta}(\mathbf{q}, \omega)= & \frac{\left(g \mu_{B}\right)^{2}}{2 \pi \hbar} \frac{1}{N} \sum_{\mathbf{R R}^{\prime}} \int d t e^{i\left[\omega t-\mathbf{q} \cdot\left(\mathbf{R}-\mathbf{R}^{\prime}\right)\right]} \\
& \times\left\langle S_{\mathbf{R}}^{\alpha}(t) S_{\mathbf{R}^{\prime}}^{\beta}(0)\right\rangle .
\end{aligned}
$$

$g$ is the Landé factor, $S_{\mathbf{R}}^{\alpha}(t)$ is the $\alpha$ th Cartesian spin component of the $\mathrm{V}$ ion at position $\mathbf{R}$ at time $t$, and \langle\rangle denotes a thermal average. When spin space anisotropy can be neglected, as in the paramagnetic phase of a Heisenberg system, expression (1) simplifies to

$$
\frac{d^{2} \sigma}{d \Omega d E^{\prime}}=2 \frac{k^{\prime}}{k}\left(\frac{\gamma r_{0}}{2}\right)^{2}[f(\mathbf{q})]^{2} e^{-2 W(\mathbf{q})} N \mathcal{S}^{\alpha \alpha}(\mathbf{q}, \omega) .
$$

We will drop the superscripts in the remaining text for brevity. The structure factor is related to the imaginary part of the dynamic spin susceptibility by the fluctuation-dissipation theorem

$$
\mathcal{S}(\mathbf{q}, \omega)=\frac{1}{1-e^{-\hbar \omega / k_{B} T}} \frac{1}{\pi} \chi^{\prime \prime}(\mathbf{q}, \omega) .
$$

The real part of the generalized spin susceptibility is given by the Kramers-Kronig relation, 


$$
\chi^{\prime}(\mathbf{q}, \omega)-\chi^{\prime}(\mathbf{q}, \infty)=\frac{1}{\pi} \int_{-\infty}^{\infty} d \omega^{\prime} \frac{\chi^{\prime \prime}\left(\mathbf{q}, \omega^{\prime}\right)}{\omega^{\prime}-\omega}
$$

The intensity in a neutron scattering experiment is measured as the ratio of neutron counts in a high efficiency ${ }^{3} \mathrm{He}$ detector to the neutron counts in a low efficiency monitor placed in the incident beam before the sample. To cover a wide energy range of spin excitations, several spectrometer configurations were used. The relative sensitivity of different configurations was determined by comparing the integrated intensities of identical scans probing magnetic or incoherent nuclear scattering. Normalizing to inelastic scattering from acoustic phonons ${ }^{56,57}$ yielded absolute measurements of $\mathcal{S}(\mathbf{q}, \omega)$ [Eq. (2)] in units of $\mu_{B}^{2} / \mathrm{meV}$. Units of mbarn $/ \mathrm{meV}$ are also used in this paper and when we do so we are quoting numbers for the following normalized intensity:

$$
\widetilde{I}(\mathbf{q}, \omega)=2\left(\frac{\gamma r_{0}}{2}\right)^{2}[f(\mathbf{q})]^{2} \mathcal{S}(\mathbf{q}, \omega),
$$

which is more directly related to the raw data because it contains the magnetic form factor, $f(\mathbf{q})$, as a factor.

\section{Resolution effects}

The measured intensity in a neutron scattering experiment is proportional to the convolution of the scattering cross section with an instrumental resolution function. The resolution function can be approximated by a Gaussian function in four-dimensional $\mathbf{q}-\omega$ space. ${ }^{58,59}$ Inelastic neutron scattering experiments are usually flux-limited. Therefore the choice of an experimental configuration for probing a specific scattering cross section always involves a trade-off between resolution and sensitivity. The following two limits are of importance in this study.

\section{Infinite lifetime excitations}

Examples of these types of excitations, such as phonons and undamped antiferromagnetic spin waves, are described by a scattering cross section of the form

$$
\mathcal{S}(\mathbf{q}, \omega) \sim \delta(\omega-c|\mathbf{q}|) .
$$

If the curvature of the dispersion surface is negligible over the volume of the resolution function, then the dispersion surface can be approximated by a flat plane and the resulting profile of a scan through the dispersion surface is approximately Gaussian with a width defined by the resolution. With coarser resolution, the profile becomes asymmetric and the peak position may shift from the position defined by the dispersion relation. Refer to the dotted lines in Fig. 10 for a few examples of such effects.

\section{Weakly q-and $\omega$-dependent scattering cross section}

Features in the scattering cross section which are broader in q- $\omega$ space than the experimental resolution are reproduced directly or with slight additional broadening in the measured q- and $\omega$-dependent intensity. This is the case for our measurements in the AFM, PM, and PI phases. For example, if $\mathcal{S}(\mathbf{q}, \omega)$ takes the form

$$
\mathcal{S}(\mathbf{q}, \omega) \sim \exp \left(-\left|\mathbf{q}-\mathbf{q}_{0}\right|^{2} / \sigma_{0}^{2}\right),
$$

a constant energy scan will yield a Gaussian peak with width $\left(\sigma_{0}^{2}+\sigma_{R}^{2}\right)^{1 / 2}$, where $\sigma_{R}$ is the resolution width. To appreciate the resolution broadening, let us put in numbers: When the resolution width is $1 / 3$ of the intrinsic width, the measured width increases by less than $10 \%$ over the intrinsic width. When the resolution width is $1 / 5$ of the intrinsic width, the measured width is only $2 \%$ larger than the intrinsic width. In this latter case the measured intensity is then very close to being directly proportional to $\mathcal{S}(\mathbf{q}, \omega)$.

\section{SDW IN METALLIC $\mathrm{V}_{2} \mathrm{O}_{3}$}

The insulating antiferromagnetic state (AFI) of $\mathrm{V}_{2} \mathrm{O}_{3}$ can be suppressed by a pressure of $\sim 20$ kbar. $^{60,49}$ The pressurestabilized metallic state is paramagnetic down to at least 0.35 $\mathrm{K} .{ }^{61,49}$ The AFI state can also be suppressed at ambient pressure by vanadium deficiency ${ }^{62}$ or titanium doping. ${ }^{19}$ The critical concentrations are $x_{c} \simeq 0.1$ for $\mathrm{V}_{2-x} \mathrm{Ti}_{x} \mathrm{O}_{3}$ (Ref. 19) and $y_{c} \simeq 0.015$ for $\mathrm{V}_{2-y} \mathrm{O}_{3} .{ }^{45}$ The existence of antiferromagnetic order at low temperatures for the doping induced metal was first established through the ${ }^{57} \mathrm{Fe}$ Mössbauer effect. ${ }^{63-65}$ The magnetic structure was only recently determined through single-crystal neutron diffraction, ${ }^{33}$ and it is depicted in Fig. 3(a). This magnetic order can be described as puckered antiferromagnetic honeycomb spin layers whose spin directions form a helix along the $c$ axis. The spin directions determined from our neutron diffraction refinement, which lie in the basal plane, are consistent with previous susceptibility anisotropy and magnetotorque measurements. ${ }^{64,66}$ The pitch of the helix yields an incommensurate magnetic structure, with magnetic wave vector $1.7 \mathbf{c}^{*}$. Both the staggered moment and the wave vector depend weakly on doping, $y{ }^{33}$ This magnetic order in metallic $\mathrm{V}_{2-y} \mathrm{O}_{3}$ bears little resemblance to the magnetic order in the insulating phase of $\mathrm{V}_{2} \mathrm{O}_{3}$ which is characterized by a magnetic wave vector $(1 / 2,1 / 2,0)^{24}$ [refer to Fig. 3(b)].

We have previously shown ${ }^{33}$ that the antiferromagnetic order in the metallic state cannot be described by a localized spin model, since the dynamic magnetic spectral weight greatly exceeds the square of the staggered moment and the bandwidth for magnetic excitations exceeds $k_{B} T_{N}$ by more than one order of magnitude. The proper concept is probably that of a spin density wave $\mathrm{e}^{67-69}$ where magnetic order results from a Fermi surface nesting instability. This interpretation is supported by recent RPA calculation of the $\mathbf{q}$-dependent susceptibility using realistic band structure. ${ }^{70}$ According to neutron, specific heat, and transport measurements, only a small fraction of a large Fermi surface ${ }^{33}$ is involved in the SDW of metallic $\mathrm{V}_{2-y} \mathrm{O}_{3}$. As seen at the SDW transition for $\mathrm{Cr}$ metal, ${ }^{71}$ the resistivity for metallic $\mathrm{V}_{2-y} \mathrm{O}_{3}$ increases below the Néel temperature as the Fermi surface is partially gapped. ${ }^{33}$ This is in contrast to localized spin systems where the resistivity decreases below $T_{N}$ because of the decrease in spin disorder scattering. The discovery of the transverse incommensurate SDW resolved the longstanding mystery ${ }^{72}$ about the magnetic ground state of doped metallic $\mathrm{V}_{2} \mathrm{O}_{3}$. 


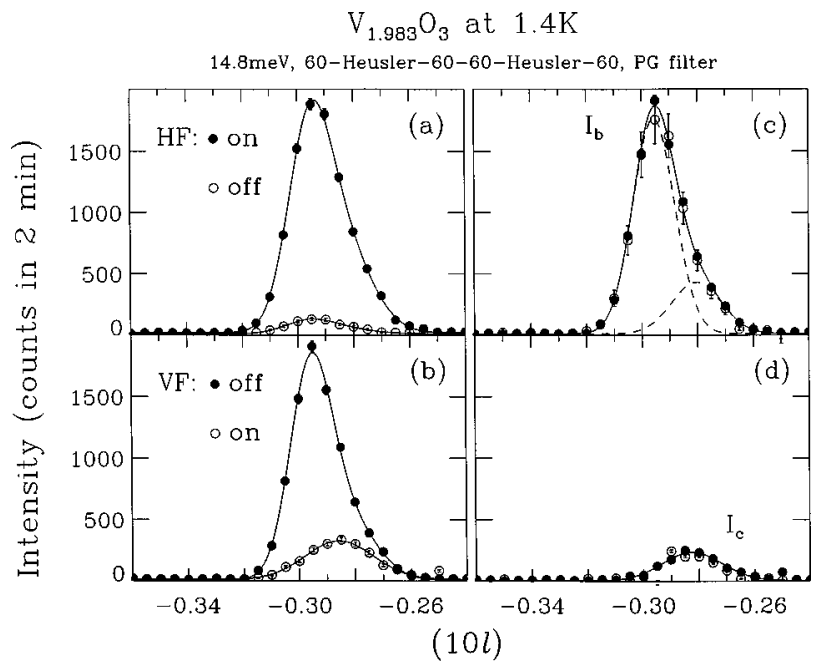

FIG. 6. Triple-axis polarized neutron scans through the $(1,0, \overline{0.3})$ magnetic Bragg peak with neutron spins polarized along the momentum transfer (HF) in (a), and along the vertical direction (VF) in (b). Measurements with the neutron spin flipper on and off are presented. The contribution to scattering from spin components in the basal plane is shown in (c) and the contribution from components polarized along the $\mathbf{c}$ axis is shown in (d). See text for details.

\section{A. Polarized neutron scattering}

To further characterize the incommensurate magnetic structure, polarized neutron measurements were performed. A $\mathrm{V}_{1.983} \mathrm{O}_{3}$ sample was oriented with its $(h 0 l)$ zone in the horizontal scattering plane. A small magnetic field of $7.6 \mathrm{G}$ was applied along either the scattering wave vector (HF) or along the vertical direction (VF) to guide the neutron spin. A neutron spin flipper was inserted in the neutron path. In the HF case when the spin flipper is turned on, only magnetic scattering contributes to the Bragg peak. ${ }^{73}$ Coherent nuclear scattering is non-spin-flip and contributes when the flipper is off. Therefore, Fig. 6(a) shows that the $(1,0, \overline{0.3})$ Bragg peak is indeed magnetic. The finite intensity in the flipper-off case is due to incomplete polarization of the neutron beam and is consistent with a flipping ratio of $f_{H}=14$, measured at nuclear Bragg peaks.

Since $0.3 c^{*} / a^{*} \approx 0.09,(1,0, \overline{0.3})$ is essentially parallel to the $\mathbf{a}^{*}$ axis. The four partial intensities at $(1,0, \overline{0.3})$ for the $\mathrm{HF}$ and VF cases with the spin flipper on or off are therefore $^{73}$

$$
\begin{gathered}
I_{\mathrm{HF}}^{\mathrm{on}}=I_{b}+I_{c}, \\
I_{\mathrm{HF}}^{\mathrm{off}}=\left(I_{b}+I_{c}\right) / f_{H}, \\
I_{\mathrm{VF}}^{\mathrm{on}}=I_{c}+I_{b} / f_{V}, \\
I_{\mathrm{VF}}^{\mathrm{off}}=I_{b}+I_{c} / f_{V},
\end{gathered}
$$

where $I_{b(c)} \propto S_{b(c)}^{2}$ is the contribution from the spin components in the $\mathbf{b} \equiv \mathbf{a}^{*} \times \mathbf{c}^{*}$ (or c) direction, and $f_{H(V)} \simeq 14$ is the flipping ratio in the HF (VF) case. Therefore, from the data shown in Figs. 6(a) and 6(b), $I_{b}$ and $I_{c}$ can be extracted by solving the overdetermined equations $(7 a)-(7 d)$. For example,

$$
\begin{gathered}
I_{b}=I_{\mathrm{VF}}^{\mathrm{off}}-I_{c} / f_{V} \approx I_{\mathrm{VF}}^{\mathrm{off}}, \\
I_{c}=\frac{I_{\mathrm{VF}}^{\mathrm{on}}-I_{\mathrm{HF}}^{\mathrm{off}}}{1-1 / f_{V}} \approx I_{\mathrm{VF}}^{\mathrm{on}}-I_{\mathrm{HF}}^{\mathrm{off}} .
\end{gathered}
$$

In the last step in Eqs. (8), a small quantity $I_{c} / f_{V}$ is neglected. Alternatively,

$$
\begin{gathered}
I_{b}=I_{\mathrm{HF}}^{\mathrm{on}}+I_{\mathrm{HF}}^{\mathrm{off}}-I_{\mathrm{VF}}^{\mathrm{on}}-I_{c} / f_{V} \approx I_{\mathrm{HF}}^{\mathrm{on}}+I_{\mathrm{HF}}^{\mathrm{off}}-I_{\mathrm{VF}}^{\mathrm{on}}, \\
I_{c}=\frac{I_{\mathrm{HF}}^{\mathrm{on}}-I_{\mathrm{VF}}^{\mathrm{off}}}{1-1 / f_{V}} \approx I_{\mathrm{HF}}^{\mathrm{on}}-I_{\mathrm{VF}}^{\mathrm{off}} .
\end{gathered}
$$

Figures 6(c) and 6(d) show the basal plane component $I_{b}$ and the vertical component $I_{c}$ derived from the data in Figs. 6(a) and 6(b) using these expressions. The solid circles were determined using Eq. (8) and the open circles using Eq. (9). The two data sets are consistent within experimental uncertainty and show that the incommensurate peak is associated with basal plane spin correlations as was previously conjectured on the basis of unpolarized neutron diffraction. The data also reveal a second incommensurate peak with mixed polarization. This peak has only been observed in this sample which is very close to the critical concentration, $y_{c}$ $\approx 0.015$, for the ambient pressure metallic phase. ${ }^{33}$

When polarized neutrons are scattered by a right-handed transverse spiral in the HF configuration, the partial cross sections for magnetic Bragg peaks with wave vectors along the spiral axis are ${ }^{73}$

$$
\frac{d \sigma(\mathbf{q})^{+\rightarrow-}}{d \Omega} \propto \sum_{\mathbf{R R}^{\prime}} e^{i\left(\mathbf{q}+\mathbf{q}_{m}\right) \cdot\left(\mathbf{R}-\mathbf{R}^{\prime}\right)} \sim \sum_{\boldsymbol{\tau}} \delta\left(\mathbf{q}+\mathbf{q}_{m}-\boldsymbol{\tau}\right)
$$

for the neutron spin-up to spin-down channel and

$$
\frac{d \sigma(\mathbf{q})^{-\rightarrow+}}{d \Omega} \propto \sum_{\mathbf{R} \mathbf{R}^{\prime}} e^{i\left(\mathbf{q}-\mathbf{q}_{m}\right) \cdot\left(\mathbf{R}-\mathbf{R}^{\prime}\right)} \sim \sum_{\boldsymbol{\tau}} \delta\left(\mathbf{q}-\mathbf{q}_{m}-\boldsymbol{\tau}\right)
$$

for the neutron spin-down to spin-up channel. In these expressions the nuclear reciprocal-lattice vector $\boldsymbol{\tau}$ and the spiral wave vector $\mathbf{q}_{m}$ are both parallel to the spiral axis. Note that only one of the two magnetic satellite Bragg peaks appears in each channel. For a left-handed spiral, the signs of $\mathbf{q}_{m}$ in these expressions reverse. Therefore, if the SDW in $\mathrm{V}_{2-\mathrm{y}} \mathrm{O}_{3}$ is a spiral with a macroscopic handedness, we should find that magnetic Bragg peaks with $\mathbf{Q}=(00 l)$ come and go with a $180^{\circ}$ rotation of the incident neutron spin state.

Figure 7 shows a double-axis polarized neutron scan through the $(0,0,7.7)$ magnetic Bragg peak of the SDW. The left panel shows raw data taken at $1.4 \mathrm{~K}$, while in the right panel a nonmagnetic background taken above the Néel temperature has been subtracted. Clearly the magnetic Bragg intensity does not change upon flipping the incident neutron spin state. Hence we conclude that the SDW phase does not have an intrinsic macroscopic handedness. This means that if the ordered phase is a spiral, our sample contains equal volume fractions of left- and right-handed spirals. Another possibility is that we have an amplitude modulated SDW which is a microscopic superposition of the left- and right-handed spirals. 


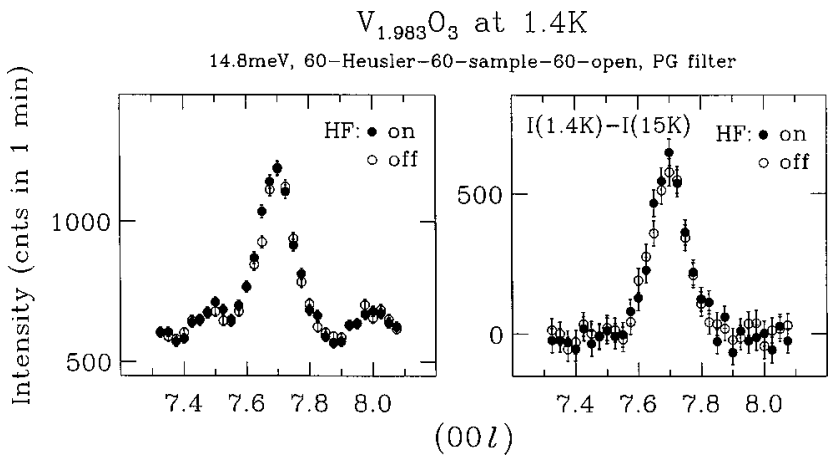

FIG. 7. (Left) Double-axis polarized neutron scans at $(0,0,7.7)$ with the neutron spin polarized along the $c$ axis (HF) at $1.4 \mathrm{~K}$. (Right) Identical scans at $15 \mathrm{~K}$ were subtracted as a measure of nonmagnetic background. The magnetic peaks are independent of the incident neutron spin state, which shows that the SDW phase has no macroscopic handedness.

\section{B. SDW, PM, and AFI phase boundary at $T=0$}

After confirming the magnetic structure in the AFM phase with polarized neutrons, let us now try to delimit the phase boundary of this SDW ground state in the pressurecomposition plane for $\mathrm{V}_{2-y} \mathrm{O}_{3}$. Two of the samples we studied with high-pressure neutron diffraction $(y=0.012$ and 0.015) have the AFI ground state at ambient pressure and display the metallic state only under pressure. Figure 8 (a) shows the $(1 / 2,1 / 2,0)$ Bragg peak which is the order parameter of the AFI, as a function of temperature at various pressures for $\mathrm{a} \mathrm{V}_{1.988} \mathrm{O}_{3}$ sample. Under high pressure, the transition to the AFI remains strongly first order, as shown here by the sudden onset of antiferromagnetic order with a nearly saturated magnetic moment.

The AFI state is completely suppressed above $4.1 \mathrm{kbar}$ for $\mathrm{V}_{1.988} \mathrm{O}_{3}$. A survey at $5 \mathrm{kbar}$ and $1.4 \mathrm{~K}$ in a quadrant of the $(h h l)$ Brillouin zone reveals no magnetic peaks associated with the AFI phase [refer to Fig. 8(b)]. On the other hand, the Bragg peaks associated with SDW order are absent for $P=4.0$ kbar but appear under a pressure of $4.6 \mathrm{kbar}$ [refer to Fig. 8(c)]. The temperature-pressure phase diagram for $\mathrm{V}_{1.988} \mathrm{O}_{3}$ is shown in Fig. 8(d). The SDW transition is second order, ${ }^{33,36}$ while the first-order AFI transition shows large thermal hysteresis.

Figure 9 summarizes our current knowledge about ground states in $\mathrm{V}_{2-y} \mathrm{O}_{3}$. For $y=0$, after the AFI is suppressed, the metallic state is paramagnetic. For $y=0.012$ and 0.015 , we found that after the AFI is suppressed, the metallic state is a SDW. Up to $8 \mathrm{kbar}$, the upper limit of our pressure cell, the SDW remains the stable low-temperature state for all the samples $(y=0.012,0.015$, and 0.027$)$ which we have studied with high-pressure neutron diffraction. ${ }^{33,36}$ By linear extrapolation of the pressure-dependent Néel temperatures, we derived estimates for the critical pressures of the SDW phase and these are indicated by open circles in Fig. 9.

Anderson has pointed out the possibility of a superconducting instability in the Brinkman-Rice liquid. ${ }^{74}$ For metallic $\mathrm{V}_{2-y} \mathrm{O}_{3}$, SDW order appears to be the dominant instability. However, it should be noted that the possibility of a superconducting state with $T_{c}<0.1 \mathrm{~K}$ at high pressures has not been excluded yet. ${ }^{75}$
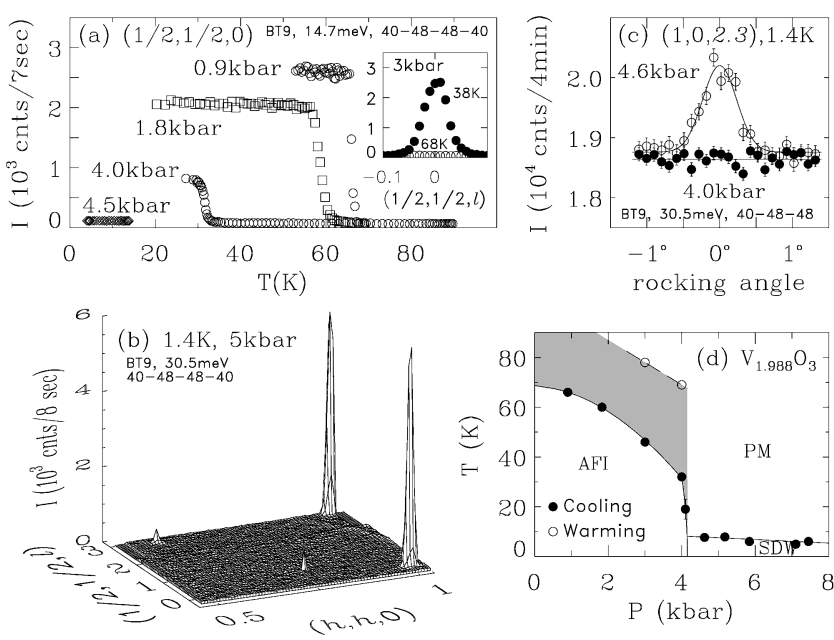

FIG. 8. Phase transitions at high pressure for $\mathrm{V}_{1.988} \mathrm{O}_{3}$. (a) Suppression of the AFI state by pressure, as measured by monitoring the $(1 / 2,1 / 2,0)$ magnetic Bragg peak upon cooling. The inset shows scans through $(1 / 2,1 / 2,0)$ at 3 kbar above (open circles) and below (solid circles) the AFI transition. (b) Elastic scans at $1.4 \mathrm{~K}$ and 5.0 kbar, covering a region in the $(h h l)$ zone slightly larger than a quadrant of the Brillouin zone. Besides two spurious peaks which were found to be temperature independent, there are only two resolution-limited nuclear Bragg peaks, (110) and (113). [No data were taken at the two corners near $(1 / 2,1 / 2,3)$ and (113).] (c) Elastic neutron scans at $1.4 \mathrm{~K}$ through $(1,0,2.3)$ under pressures which stablize the AFI (solid circles) and the SDW (open circles) ground states, respectively. (d) The pressure-temperature phase diagram for $\mathrm{V}_{1.988} \mathrm{O}_{3}$. The hatched region shows the thermal hysteresis at this first-order transition.

\section{SPIN EXCITATIONS AND QUANTUM CRITICAL BEHAVIOR IN METALLIC PHASES}

\section{A. Stoner electron-hole pair continuum at $T<T_{N}$}

Figure 10 shows constant energy transfer, $\hbar \omega$, scans spanning a whole Brillouin zone in the AFM phase for $\mathrm{V}_{1.973} \mathrm{O}_{3}\left[T_{N}=8.5(1) \mathrm{K}\right]$. The incommensurate Bragg

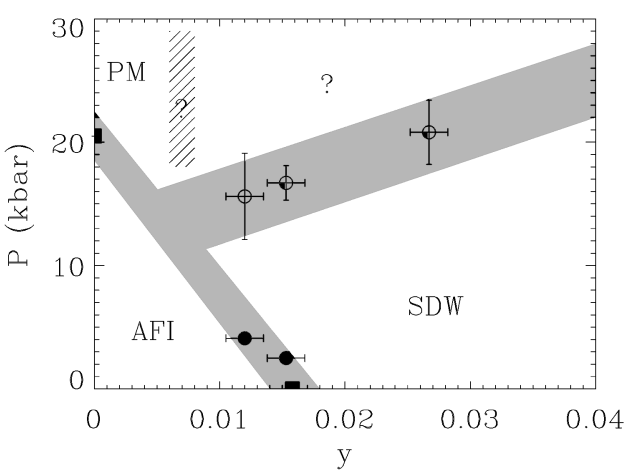

FIG. 9. The composition-pressure phase diagram at $T=0$ for $\mathrm{V}_{2-y} \mathrm{O}_{3}$. The squares are from Refs. 60 and 45 and circles are from the current neutron mesurement. Open circles are extrapolated from data below 8 kbar. For $y \sim 0$, the paramagnetic metallic (PM) state is stable at high pressures. However, the extent of the PM state beyond the hatched region and down to temperatures below $0.1 \mathrm{~K}$ is not clear. It has not been possible to produce single phase samples of $\mathrm{V}_{2-\mathrm{y}} \mathrm{O}_{3}$ for $y>0.05$ because of the formation of $\mathrm{V}_{3} \mathrm{O}_{5}$ (Refs. 63 and 44). 


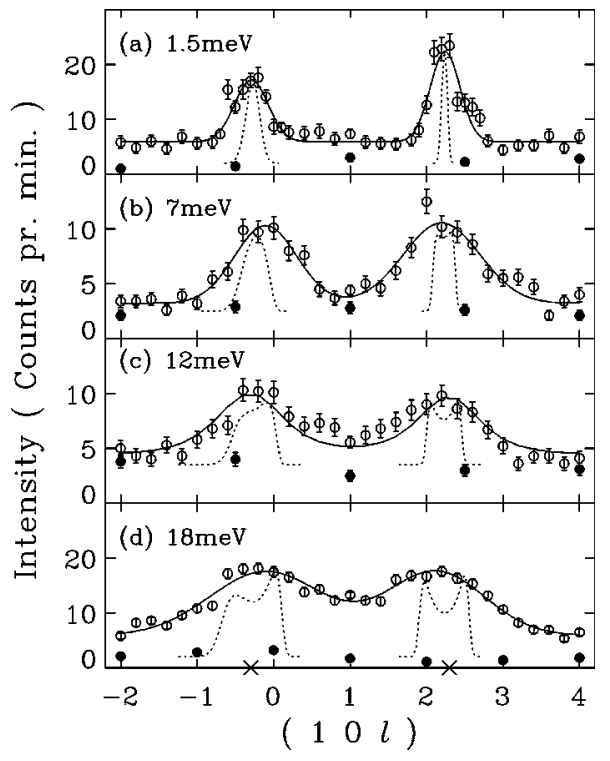

FIG. 10. Constant energy, $\hbar \omega$, scans at $1.5 \mathrm{~K}$ for $\mathrm{V}_{1.973} \mathrm{O}_{3}$ across a Brillouin zone. (10 $\overline{2})$ and (104) are two nuclear Bragg points, and $(1,0, \overline{0.3})$ and $(1,0,2.3)$ are two incommensurate magnetic satellites (marked by crosses). The spectrometer configurations at BT2 were $E_{f}=13.7 \mathrm{meV}$ with horizontal collimations $60^{\prime}-40^{\prime}-40^{\prime}-40^{\prime}$ for (a) $-(\mathrm{c})$, and $E_{i}=35 \mathrm{meV}$ with $60^{\prime}-40^{\prime}-60^{\prime}-60^{\prime}$ for (d). Vertical collimations were $300^{\prime}-170^{\prime}-230^{\prime}-970^{\prime}$ in all four cases. The filled circles are analyzer turned backgrounds. The dotted lines represent the fourdimensional convolutions of the instrumental resolution with an isotropic spin wave dispersion relation with a velocity $c$ $=130 \mathrm{meV} \AA$.

points, $(1,0, \overline{0.3})$ and $(1,0,2.3)$, covered by these scans correspond to the long-range ordered SDW. The solid circles represent a flat background measured by turning the PG(002) analyzer $10^{\circ}$ away from the reflection condition. Around each magnetic Bragg point, $(1,0, \overline{0.3})$ or $(1,0,2.3)$, there is only one broad peak. This is not due to poor resolution merging two counterpropagating spin wave modes: the dotted lines in the figure represent the calculated spectrometer response to spin waves with a velocity $c=130 \mathrm{meV} \AA$, which is much larger than the inverse slope of the peak width versus energy transfer curve (see Fig. 11). Our resolution is clearly adequate to rule out a conventional spin wave response in this energy range. Therefore the broad intensity profile which we observed is intrinsic, and in the energy range probed, magnetic excitations consist of a single broad lobe at each magnetic Bragg point.

For insulating magnets, propagating spin waves exist over the whole Brillouin zone. For itinerant magnets, however, propagating spin waves only exist in a limited range near the magnetic Bragg peaks. Beyond this range they merge into the Stoner particle-hole continuum. According to weak coupling theory, ${ }^{69}$ the energy gap which a SDW creates inside the particle-hole continuum is $\Delta=1.76 \alpha k_{B} T_{N}$, where $\alpha$ $\equiv\left(v_{1}+v_{2}\right) / 2\left(v_{1} v_{2}\right)^{1 / 2}$ and $v_{1}$ and $v_{2}$ are Fermi velocities of the two nesting bands. Inside this gap, spin waves propagate with a velocity ${ }^{69,76,77} c=\left(v_{1} v_{2} / 3\right)^{1 / 2}$. For metallic $\mathrm{V}_{2-y} \mathrm{O}_{3}$ at $T=0$, spin waves are expected only below $\sim 1.76 k_{B} T_{N}$ $=1.4 \mathrm{meV}$, which is below the energies which we have

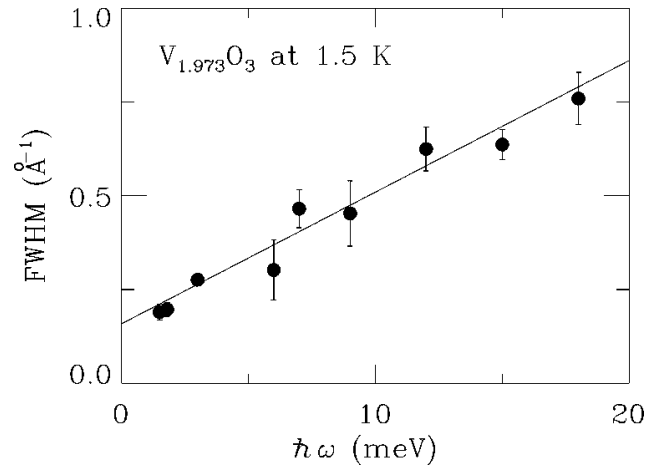

FIG. 11. Resolution-corrected peak widths (FWHM) of constant energy scans as a function of energy transfer for $\mathrm{V}_{1.973} \mathrm{O}_{3}$ in the long-range-ordered SDW state at $1.5 \mathrm{~K}$.

probed in this experiment. The broad lobes measured in Fig. 10 thus represent magnetic excitations inside the Stoner continuum.

Other signs that we are probing magnetic fluctuations in the electron-hole continuum, as opposed to transverse oscillations of the order parameter, are (i) the spectral weight from $1.5 \mathrm{meV}$ to $18 \mathrm{meV}$ in Fig. 10 corresponds to a fluctuating moment of $0.32 \mu_{B}$ per $\mathrm{V}$, already twice that of the static staggered moment, ${ }^{33}$ and (ii) the resolution corrected full widths at half maximum (FWHM) of constant energy scans (see Fig. 11) extrapolate to a finite value at $\omega=0$, even though there are true, resolution-limited Bragg peaks at $\omega$ $=0$.

For Cr metal, ${ }^{78,79}$ another 3D SDW $\left(T_{N}=310 \mathrm{~K}\right)$, one would expect at $T=0$ that spin wave modes merge into a single broad lobe above $\sim 1.76 k_{B} T_{N}=47 \mathrm{meV}$. However, due to the much larger spin wave velocity in $\mathrm{Cr}$, estimated at $\sim 1000 \mathrm{meV} \AA,{ }^{80,81}$ it has not yet been possible in this material to distinguish between resolution merged spin waves and single lobe excitations. We were aided in the case of metallic $\mathrm{V}_{2} \mathrm{O}_{3}$ by strong correlations. The enhanced effective mass reduces the Fermi velocity to a value which is $\sim 1 / 15$ of that for $\mathrm{Cr}^{33}$ This makes it feasible to unambiguously observe these unique single-lobe magnetic excitations in the SDW state of $\mathrm{V}_{2-y} \mathrm{O}_{3}$.

\section{B. Doping effects in the metallic phases}

We have previously shown ${ }^{33}$ that both the staggered moment and the incommensurate wave vector of the static SDW order depend only weakly on doping. For a change in metal deficiency $\delta y=0.03$ which is twice the critical $y_{c}$ for the AFI phase, the change in the magnetic wave vector $\left(1.7 \mathbf{c}^{*}\right)$ is only $\sim 0.02 \mathbf{c}^{*}$. Likewise, dynamic magnetic correlations in the PM phase are insensitive to doping. As shown in Fig. 12, even for pure $\mathrm{V}_{2} \mathrm{O}_{3}$, which has a different low-temperature AFI state, the magnetic response at $200 \mathrm{~K}$ is indistinguishable from that of $\mathrm{V}_{1.973} \mathrm{O}_{3}$. In particular, we do observe peaks at the SDW wave vectors. This demonstrates that magnetism throughout the metallic phase is controlled by the same Fermi surface instability and indicates that small moment incommensurate antiferromagnetism in $\mathrm{V}_{2-\mathrm{y}} \mathrm{O}_{3}$ is not due to impurity effects but is an intrinsic property of metallic $\mathrm{V}_{2} \mathrm{O}_{3}$. 


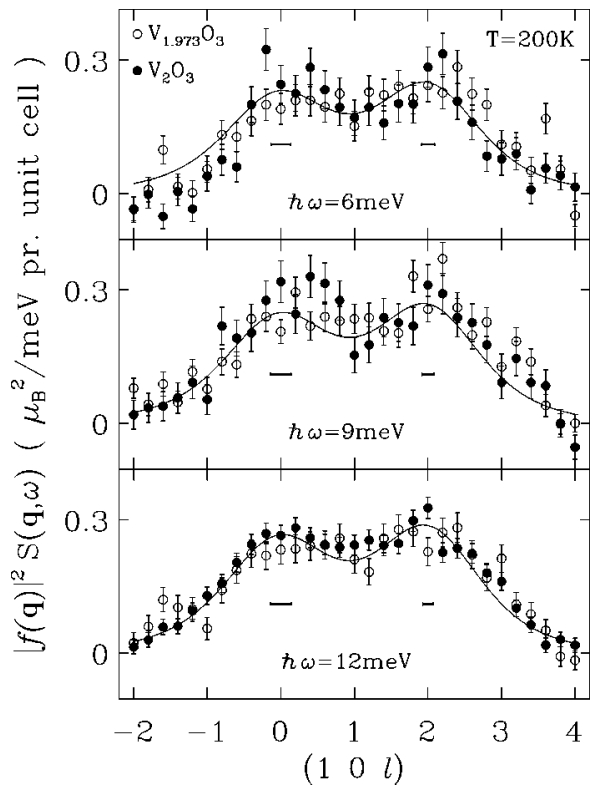

FIG. 12. Magnetic responses at $200 \mathrm{~K}$ from $\mathrm{V}_{1.973} \mathrm{O}_{3}$ (open circles) and $\mathrm{V}_{2} \mathrm{O}_{3}$ (solid circles) in the PM phase. Although these two samples have different low-temperature states, the SDW for the former and the AFI for the latter, there is no difference in the dynamic correlations in the PM phase. The experimental configuration was $E_{f}=13.7 \mathrm{meV}$ with horizontal collimations $60^{\prime}-40^{\prime}-40^{\prime}-60^{\prime}$ for $\mathrm{V}_{1.973} \mathrm{O}_{3}$ at BT2. The horizontal bars indicate the FWHM of the projection of the resolution function on the scan direction. The experimental configurations for $\mathrm{V}_{2} \mathrm{O}_{3}$ were $\mathrm{E}_{f}$ $=13.7 \mathrm{meV}$ with horizontal collimations $40^{\prime}-60^{\prime}-80^{\prime}-80^{\prime}$ for $\hbar \omega=6$ and $9 \mathrm{meV}$, and $E_{i}=35 \mathrm{meV}$ with $40^{\prime}-40^{\prime}-80^{\prime}-80^{\prime}$ for 12 $\mathrm{meV}$ at BT4 with PG monochromator and analyzer. The solid line is a least-squares fit to Eqs. (19) and (18).

\section{Antiferromagnetic quantum critical behavior in metal}

The SDW in metallic $\mathrm{V}_{2-y} \mathrm{O}_{3}$ is a small moment, lowtemperature antiferromagnetic ordering. The staggered moment in the magnetic ground state $\left(0.15 \mu_{B}\right)$ is only a small fraction of the effective paramagnetic moment $\left(2.37-2.69 \mu_{B}\right)$ which appears in the high-temperature Curie-Weiss susceptibility. ${ }^{82,83,66}$ In other words, the magnetic system is very close to a quantum critical point. ${ }^{84-87}$ We have previously shown ${ }^{36}$ that the single lobe spin excitation spectrum in metallic $\mathrm{V}_{1.973} \mathrm{O}_{3}$ is isotropic in space, and the spectrum $\chi^{\prime \prime}(\mathbf{q}, \omega)$, from $1.4 \mathrm{~K}$ inside the ordered phase to temperatures above $20 T_{N}$, has been determined in absolute units. ${ }^{88}$ Here we discuss our results in connection with the quantum critical behavior.

Figure 13(a) shows in a log-log scale the inverse correlation length, $\kappa$, measured in Ref. 36 as a function of the reduced temperature, $t \equiv\left(T-T_{N}\right) / T_{N}$. The solid line is $\kappa$ $\propto t^{\nu}$ with $\nu=0.55(4)$ from a least-squares fit. This value of $\nu$ suggests a small positive $\eta$ exponent according to the scaling relation $^{89} \eta=2-\gamma / \nu$. However, the classical values of $\nu$ $=1 / 2, \eta=0$ are also consistent with our data (refer to the dotted line).

The spin fluctuation spectrum $\chi^{\prime \prime}(\mathbf{q}, \omega)$ reaches its maximum value, $\chi_{\max }^{\prime \prime}$, at $\omega=\omega_{p}$ and $\mathbf{q}=\mathbf{Q}$ which is an antiferromagnetic Bragg point. ${ }^{36}$ The dominant part of the spectral weight, therefore, falls in the hydrodynamic region, ${ }^{90}$ $|\mathbf{q}-\mathbf{Q}| \ll \kappa$, where $\hbar \omega_{p}=\gamma_{A} \kappa^{z}$. In Fig. 13(b), we find the
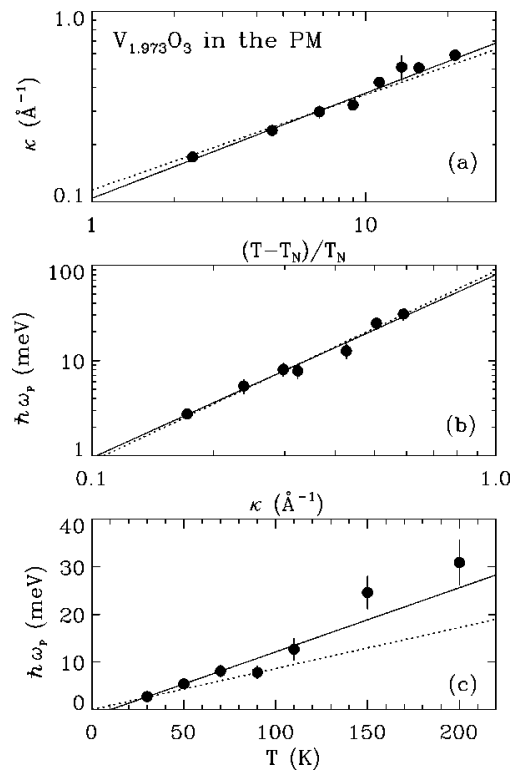

FIG. 13. (a) The inverse correlation length as a function of the reduced temperature for $\mathrm{V}_{1.973} \mathrm{O}_{3}$ in the PM phase. Both axes use the logarithmic scale. The solid line is the best fit of $\kappa \propto t^{\nu}$ to the data with $\nu=0.55(4)$, and the dotted line is a fit with fixed $\nu$ $=1 / 2$. (b) The characteristic spin fluctuation energy, $\hbar \omega_{p}$, where $\chi^{\prime \prime}(\mathbf{Q}, \omega)$ is maximized, as a function of $\kappa$. The solid line is the best fit of $\omega_{p} \propto \kappa^{z}$ to the data with $z=1.9(1)$, and the dotted line is a fit with fixed $z=2$. (c) $\hbar \omega_{p}$ is roughly proportional to the thermal energy $k_{B} T$ (the dotted line). The solid line is $\omega_{p} \propto T-T_{N}$.

dynamic exponent $z=1.9(1)$ (the solid line), which is in agreement with the theoretical prediction $z=2$ for itinerant antiferromagnets in the quantum critical region. ${ }^{84,87}$ The constant prefactor is $\gamma_{A}=87(4) \mathrm{meV}^{2}$.

Combining the scaling relations from Figs. 13(a) and 13(b), we have $\hbar \omega_{p} \sim t^{z \nu}=C k_{B}\left(T-T_{N}\right)$ [refer to the solid line in Fig. 13(c)]. Given the small value of $T_{N}$ and the fact that $C \approx 1$, the characteristic spin fluctuation energy $\hbar \omega_{p}$ is close to $k_{B} T$ [refer to the dotted line in Fig. 13(c)]. This feature is shared with nearly metallic cuprates ${ }^{91}$ and some heavy fermion systems. ${ }^{92}$ Antiferromagnetic spin fluctuations for systems close to a quantum critical point, therefore, might provide a microscopic mechanism for marginal Fermi liquid phenomenology. ${ }^{93}$

The peak intensity of some constant energy scans is shown in Fig. 14(a) as a function of energy. They are expressed in absolute units and represent $\chi^{\prime \prime}(\mathbf{Q}, \omega)$ at the magnetic zone center. At $1.5 \mathrm{~K}, \chi^{\prime \prime}(\mathbf{Q}, \omega)$ is a decreasing function of $\omega$ in the measured energy range and it changes to an increasing function at elevated temperatures. They were scaled onto a single universal curve in Fig. 14(b) by dividing $\chi^{\prime \prime}(\mathbf{Q}, \omega)$ by $\chi_{\max }^{\prime \prime}$ and $\hbar \omega$ by the peak energy $\hbar \omega_{p}$. The apparently different behaviors in a finite energy window in Fig. 14(a), therefore, reflect different segments of a universal behavior

For a general wave vector, $\mathbf{q}$, away from an antiferromagnetic Bragg point, $\mathbf{Q}$, we have shown ${ }^{36}$ previously that our neutron scattering data measured at various temperatures can be described by

$$
\frac{\chi^{\prime \prime}(\mathbf{Q}+\mathbf{q}, \omega)}{2 \chi_{\max }^{\prime \prime}}=\Phi\left(\frac{q}{\kappa}, \frac{\hbar \omega}{\gamma_{A} \kappa^{2}}\right),
$$



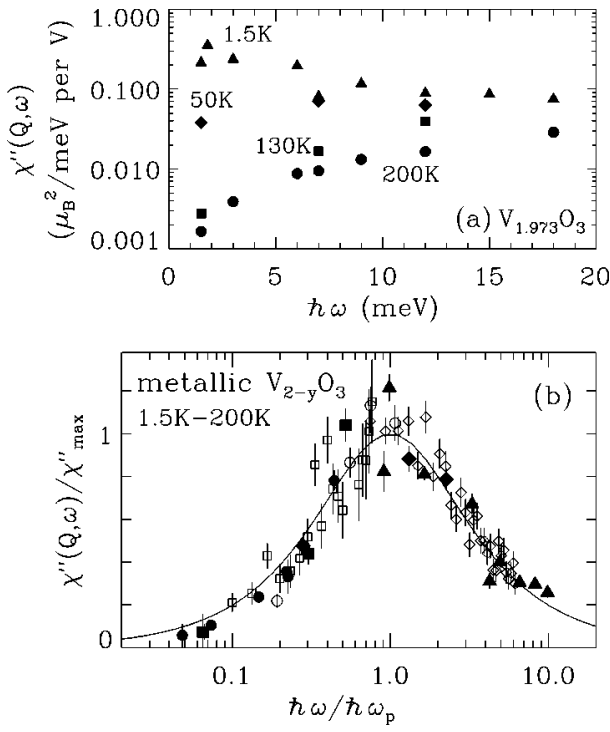

FIG. 14. (a) Normalized peak intensity of constant energy scans at $1.5 \mathrm{~K}$ (triangles), $50 \mathrm{~K}$ (diamonds), $130 \mathrm{~K}$ (squares), and $200 \mathrm{~K}$ (circles) for $\mathrm{V}_{1.973} \mathrm{O}_{3}$. In the measured energy range, $\chi^{\prime \prime}(\mathbf{Q}, \omega)$ changes from a decreasing function of $\hbar \omega$ at $1.5 \mathrm{~K}$ to an increasing function at $200 \mathrm{~K}$. (b) Data in (a) are scaled onto a universal curve using measured (Ref. 36) $\chi_{\max }^{\prime \prime}=\chi_{\mathbf{Q}} / 2$ and $\hbar \omega_{p}=\gamma_{A} \kappa^{2}$. The open circles are from constant $\hbar \omega=1.5 \mathrm{meV}$ scans at $9 \mathrm{~K}, 19 \mathrm{~K}, 27 \mathrm{~K}$, and $82 \mathrm{~K}$; the open diamonds are from a constant $\mathbf{Q}=(1,0,2.3)$ scan at $30 \mathrm{~K}$. Constant- $\mathbf{Q}$ scan for $\mathrm{V}_{2} \mathrm{O}_{3}$ at $200 \mathrm{~K}$ is shown with the open squares. The solid line is $z=2 y /\left(y^{2}+1\right)$ [refer to Eqs. (12) and (13)].

where the scaling function is

$$
\Phi(x, y)=y /\left[y^{2}+\left(1+x^{2}\right)^{2}\right] .
$$

The solid line in Fig. 14(b) is $2 \Phi(0, y)$. The maximum $\chi_{\max }^{\prime \prime}$ is related to static staggered susceptibility. ${ }^{36}$ Combining the Kramers-Kronig relation and Eq. (12) gives

$$
\begin{aligned}
\chi_{\mathbf{Q}} & \equiv \pi^{-1} \int_{-\infty}^{\infty} d \omega \chi^{\prime \prime}(\mathbf{Q}, \omega) / \omega \\
& =\chi_{\max }^{\prime \prime} 2 \pi^{-1} \int_{-\infty}^{\infty} \Phi\left(0, \hbar \omega / \gamma_{A} \kappa^{2}\right) d \omega / \omega \\
& =\chi_{\max }^{\prime \prime} 2 \pi^{-1} \int_{-\infty}^{\infty} \Phi(0, y) d y / y \\
& =\chi_{\text {max }}^{\prime \prime} \times \text { const. }
\end{aligned}
$$

Notice that when $T_{N} \rightarrow 0, \hbar \omega_{p}=C k_{B} T, \chi_{\max }^{\prime \prime} \sim \chi_{\mathbf{Q}}=a_{1} T^{-\gamma}$, and $\kappa=T^{\nu} / a_{2}$, hence Eq. (12) becomes

$$
\chi^{\prime \prime}(\mathbf{Q}+\mathbf{q}, \omega)=\frac{a_{1}}{T^{\gamma}} \Phi\left(\frac{a_{2} q}{T^{\nu}}, \frac{\hbar \omega}{C k_{B} T}\right),
$$

where $a_{1}$ and $a_{2}$ are nonuniversal constants. ${ }^{94}$ Using $z \nu \approx 1$ from our measurement and the scaling relation $\gamma=(2$ $-\eta) \nu$, and absorbing the constant $C$ in the scaling function,

$$
\chi^{\prime \prime}(\mathbf{Q}+\mathbf{q}, \omega)=\frac{a_{1}}{T^{(2-\eta) / z}} \Phi_{1}\left(\frac{a_{2} q}{T^{1 / z}}, \frac{\hbar \omega}{k_{B} T}\right) .
$$

This expression, which is supported by our experimental data, is identical to the scaling form proposed by Sachdev and $\mathrm{Ye}$ in the quantum critical region. ${ }^{86}$

\section{Theory of itinerant magnetism}

Itinerant magnetic systems have been studied within the random-phase approximation (RPA), which provides a reasonable description at $T=0$ for the ordered moment and for spin excitations in the spin wave modes and in the Stoner continuum. But it does not relate the low-temperature excitation parameters to finite-temperature properties such as the ordering temperature and the high-temperature Curie-Weiss susceptibility.

The imaginary part of the RPA generalized susceptibility for an itinerant antiferromagnet, as was done to describe magnetic fluctuations in chromium, ${ }^{76,77}$ can be written as follows:

$$
\chi^{\prime \prime}(\mathbf{Q}+\mathbf{q}, \omega)=\frac{\chi_{0} /\left(\kappa \xi_{0}\right)^{2}}{1+(q / \kappa)^{2}} \frac{\hbar \omega \Gamma(q, \kappa)}{(\hbar \omega)^{2}+\Gamma(q, \kappa)^{2}},
$$

where the wave-vector-dependent relaxation rate,

$$
\Gamma(q, \kappa)=\gamma_{A}\left(\kappa^{2}+q^{2}\right),
$$

corresponds to the simplest functional form consistent with the dynamical scaling hypothesis with a dynamic exponent $z=2$. As a comparison, for the insulating Heisenberg antiferromagnet, ${ }^{90,97} z=3 / 2$. The uniform noninteracting susceptibility $\chi_{0}$, the length scale $\xi_{0}$, and $\gamma_{A}$ are temperatureindependent parameters, and the inverse correlation length $\kappa$ approaches zero when the Néel temperature is approached. This functional form and derivations thereupon ${ }^{98}$ offer a good description of critical spin fluctuations for $\mathrm{Cr}$ and its alloys. ${ }^{81,99}$ They have also been successfully used to describe spin dynamics as measured by neutron scattering for $\mathrm{La}_{1.86} \mathrm{Sr}_{0.14} \mathrm{CuO}_{2}$ above the superconducting transition temperature. ${ }^{100} \mathrm{~A}$ modified form was used to model nuclear magnetic resonance (NMR) data in other high-temperature superconducting cuprates. ${ }^{101-103}$ However, the RPA parameters $\gamma_{0}, \chi_{0}$, and $\xi_{0}$ in Eq. (17) are free parameters which are not connected to other physically measurable quantities.

The SCR theory, ${ }^{14-18}$ a mode-mode coupling theory improves upon the RPA theory by considering self-consistently the effects of spin fluctuations on the thermal equilibrium state. It is solvable in the small moment limit with a few experimentally measurable parameters. The ferromagnetic version of the SCR theory was discussed in Refs. 14-17 and the antiferromagnetic version in Refs. 18 and 95.

The generalized susceptibility for antiferromagnets in the SCR theory ${ }^{95}$ has the same functional form as in the RPA theory:

$$
\chi^{\prime \prime}(\mathbf{Q}+\mathbf{q}, \omega)=\frac{\chi_{\mathbf{Q}}}{1+(q / \kappa)^{2}} \frac{\hbar \omega \Gamma(q, \kappa)}{(\hbar \omega)^{2}+\Gamma(q, \kappa)^{2}},
$$

where $\Gamma(q, \kappa)$ is defined as in Eq. (18). The RPA parameter $\chi_{0} /\left(\kappa \xi_{0}\right)^{2}$ is substituted now by the static staggered susceptibility $\chi_{\mathbf{Q}}$, consistent with the Kramers-Kronig relation. The major difference from the RPA theory, however, is that the SCR theory can predict other physical quantities using pa- 


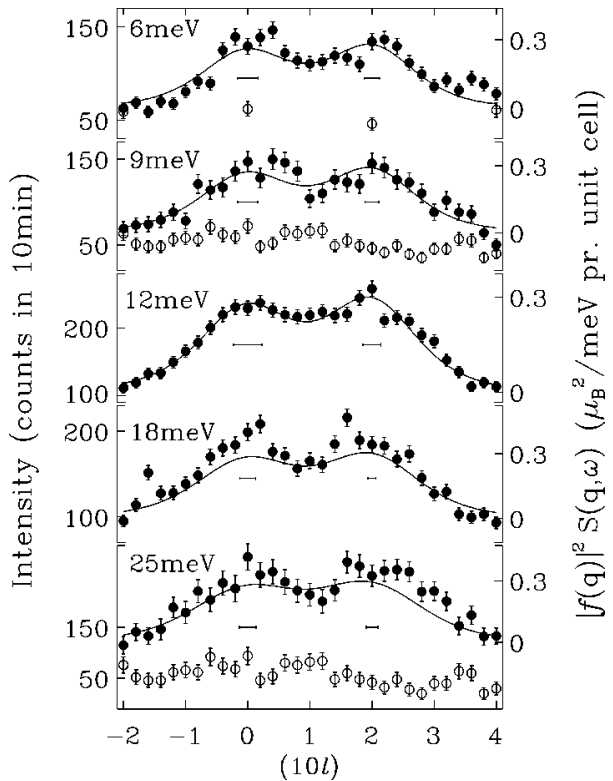

FIG. 15. Constant energy scans at $200 \mathrm{~K}$ in the PM phase (solid circles) and at $160 \mathrm{~K}$ in the AFI phase (open circles) for stoichiometric $\mathrm{V}_{2} \mathrm{O}_{3}$. Magnetic correlations near the SDW wave vectors, represented by the two peaks, disappear when the material enters the AFI phase. The experimental configurations at BT4 were $E_{f}$ $=13.7 \mathrm{meV}$ with horizontal collimations $40^{\prime}-60^{\prime}-80^{\prime}-80^{\prime}$ for $\hbar \omega=6$ and $9 \mathrm{meV}, E_{i}=35 \mathrm{meV}$ with $40^{\prime}-40^{\prime}-80^{\prime}-80^{\prime}$ for 12 and $18 \mathrm{meV}$, and $E_{f}=30 \mathrm{meV}$ with $60^{\prime}-60^{\prime}-60^{\prime}-60^{\prime}$ for $25 \mathrm{meV}$. We used PG (002) as a monochromator and analyzer except for the last configuration where a $\mathrm{Cu}(220)$ monochromator was used to improve the energy resolution. The horizontal bars indicate the FWHM of the projection of the resolution function on the scan direction. The solid line is a least-squares fit to Eqs. (19) and (18) and absolute units are given on the right scales.

rameters from the spin excitation spectrum. ${ }^{96}$ For example, the nonuniversal parameters $a_{1}$ and $a_{2}$ (Ref. 94) defined above Eq. (15) can be used to quantitatively relate $T_{N}$ to the staggered moment $M_{\mathbf{Q}}$ (refer to Fig. 4 of Ref. 36).

Figure 15 shows an example of a simultaneous fit of constant energy scans to Eqs. (19) and (18) for stoichiometric $\mathrm{V}_{2} \mathrm{O}_{3}$ at $200 \mathrm{~K}$. Solid circles represent the magnetic response in the paramagnetic metallic phase, which peaks around the SDW wave vectors. The resolution widths are much smaller than the measured widths, therefore resolution broadening can be ignored in this case. The solid line through these 200 $\mathrm{K}$ data is the best-fit functional form for $|f(\mathbf{q})|^{2} \mathcal{S}(\mathbf{q}, \omega)$ calculated using copies of Eq. (19) summed over the Bragg points $\mathbf{Q}=(1,0,4-\Delta)$ and $(1,0, \overline{2}+\Delta)$ with $\Delta=1.95(6) c^{*}$, $\kappa=1.32(5) c^{*}, \quad \gamma_{A}=18(2) \mathrm{meV} / c^{* 2}, \quad$ and $\chi_{\mathbf{Q}}$ $=0.071(7) \mu_{B}^{2} / \mathrm{meV}$ per vanadium. These values are within the error bars of those for $\mathrm{V}_{1.973} \mathrm{O}_{3},{ }^{36}$ as should be expected given the close similarity of the raw data for these two samples which are shown in Fig. 12.

It is customary to use the Lorentzian relaxation energy $\Gamma(q, \kappa)$ of Eqs. (17) or (19) as the energy scale of spin fluctuations. For an itinerant antiferromagnet close to a quantum critical point, such as metallic $\mathrm{V}_{2-y} \mathrm{O}_{3}$, this reduces to $\gamma_{A} \kappa^{2}$ at $q=0 .{ }^{36}$ To demonstrate the prominence of this energy, we plot in Fig. 16(a) the intensity contour of $\chi^{\prime \prime}(\mathbf{Q}+\mathbf{q}, \omega) / \chi_{\mathbf{Q}}$ $=\Phi\left(q / \kappa, \hbar \omega / \gamma_{A} \kappa^{2}\right)$ for small moment itinerant antiferro-
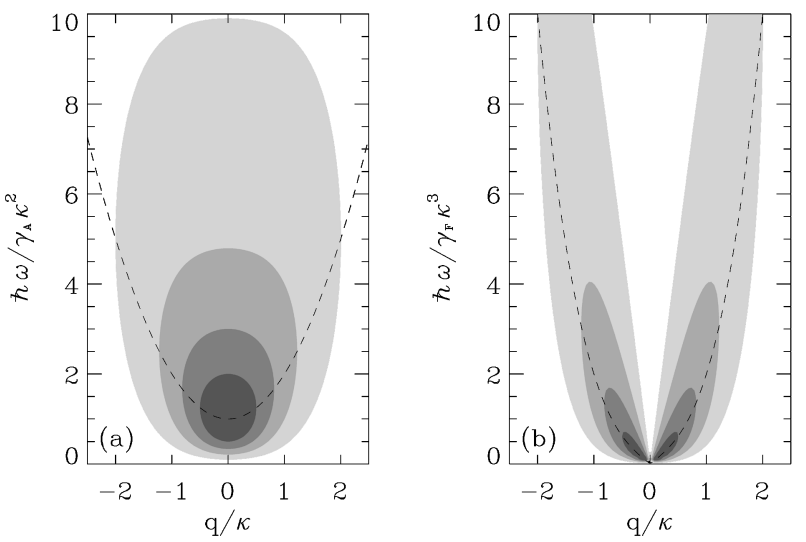

FIG. 16. Intensity contour of the scaled magnetic response function $\chi^{\prime \prime}(\mathbf{Q}+\mathbf{q}, \omega) / \chi_{\mathbf{Q}}$ as a function of reduced wave vector and reduced energy for (a) itinerant antiferromagnets and for (b) itinerant ferromagnets near a quantum critical point. The contour levels are $0.1,0.2,0.3$ and 0.4 . In the antiferromagnetic case (a), the peak intensity is 0.5 at $q=0$ and $\hbar \omega / \gamma_{A} \kappa^{2}=1$. In the ferromagnetic case (b), the intensity is singular at $q=0$ and $\omega=0$. Both $\kappa$ and $\chi_{\mathrm{Q}}$ are functions of temperature. $\gamma_{A}$ and $\gamma_{F}$ are temperature-insensitive constants. See text for details.

magnets [refer to Eqs. (19), (18), and (13)]. The spectrum is unique in that the electron-hole damping has reduced the excitation spectrum to a single lobe at the magnetic zone center, as we observed in our neutron scattering experiment for metallic $\mathrm{V}_{2-y} \mathrm{O}_{3} .{ }^{33,37}$ The peak intensity occurs at $q$ $=0$ and $\hbar \omega=\gamma_{A} \kappa^{2}$, and $\gamma_{A} \kappa^{2} \sim t^{z \nu}$ clearly is the characteristic energy scale. In this case, $z \nu \approx 1$, as we found experimentally, is essential for achieving the quantum critical scaling of Eq. (16).

This is quite different from the well studied case of small moment itinerant ferromagnets. The $\chi^{\prime \prime}(\mathbf{q}, \omega)$ predicted by the SCR theory for ferromagnets ${ }^{104,105}$ has the same Lorentzian form as Eq. (19), but the relaxation energy is given by

$$
\Gamma(q, \kappa)=\gamma_{F} q\left(\kappa^{2}+q^{2}\right) .
$$

Like the antiferromagnetic SCR theory, the ferromagnetic SCR theory not only correctly describes the spin fluctuation spectrum, but also quantitatively relates the spectrum parameters to various other physical quantities, as demonstrated in the 3D small-moment ferromagnets MnSi (Refs. 57, 105 and 106) and $\mathrm{Ni}_{3} \mathrm{Al}{ }^{104}$ Recasting Eqs. (19) and (20) in a dimensionless form,

$$
\chi^{\prime \prime}(\mathbf{Q}+\mathbf{q}, \omega) / \chi_{\mathbf{Q}}=\Psi\left(\frac{q}{\kappa}, \frac{\hbar \omega}{\gamma_{F} \kappa^{3}}\right),
$$

with

$$
\Psi(x, y)=|x| y /\left[y^{2}+\left(x+x^{3}\right)^{2}\right] .
$$

It is depicted in Fig. 16(b). Although the counterpropagating spin wave branches are over-damped by electron-hole excitations, they remain two separate branches. The intensity is singular at $q=0$ and $\omega=0$, and the Lorentzian relaxation energy (the dashed line) serves well as the energy scale in this case, as customarily performed. 

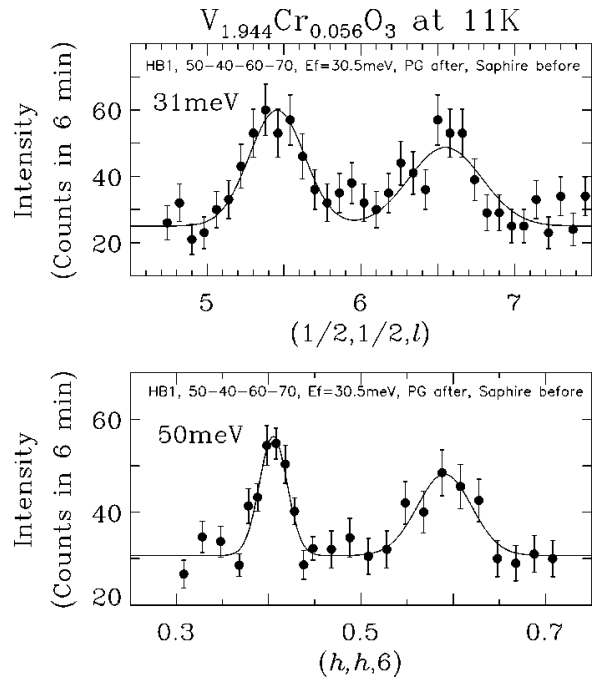

FIG. 17. Constant energy transfer scans along the (001) and (110) directions around the magnetic Bragg point $(1 / 2,1 / 2,0)$. The two peaks in each frame correspond to counterpropagating spin wave modes. The different peak shapes are due to resolution effects (focusing and defocusing).

\section{SPIN WAVES IN THE AFI PHASE}

While magnetic excitations in the metallic phase are dominated by overdamped modes, the AFI phase exhibits conventional propagating spin wave excitations. Word et al. $^{31}$ measured spin waves in this phase around $(1 / 2,1 / 2,0)$ at $142 \mathrm{~K}$ in pure $\mathrm{V}_{2} \mathrm{O}_{3}$. There is a spin excitation gap of 4.8 $\mathrm{meV}$, which is essentially independent of temperature for $T$ $<T_{N} \cdot{ }^{32}$ For $\mathrm{V}_{1.94} \mathrm{Cr}_{0.06} \mathrm{O}_{3}\left(T_{N}=177 \mathrm{~K}\right)$, the gap energy at $(1 / 2,1 / 2,0)$ is not different from that seen for the pure sample for temperatures below $50 \mathrm{~K} .{ }^{32}$ However, it shows substantial softening with rising temperature, consistent with the trend towards a continuous phase transition with increasing Cr doping.

Here we present spin waves measured at $11 \mathrm{~K}$ for $\mathrm{V}_{1.944} \mathrm{Cr}_{0.056} \mathrm{O}_{3}$. Figure 17 shows constant energy scans near a magnetic zone center. The spin wave dispersion relation has been measured for energies up to $50 \mathrm{meV}$ (see Fig. 18), which covers about one-quarter of the Brillouin zone. For comparison, some of the previous data for pure $\mathrm{V}_{2} \mathrm{O}_{3}$ are also included in Fig. 18. At low measurement temperatures and for $\hbar \omega<25 \mathrm{meV}$, there is no difference in the spin wave dispersion for pure and $3 \% \mathrm{Cr}$ doped $\mathrm{V}_{2} \mathrm{O}_{3}$.

Spin waves in the isomorphous corundum antiferromagnets $\mathrm{Cr}_{2} \mathrm{O}_{3}$ and $\alpha-\mathrm{Fe}_{2} \mathrm{O}_{3}$ have been measured over the whole Brillouin zone. ${ }^{107,108}$ These two compounds have different magnetic structures. ${ }^{109,110}$ Linear spin wave theory ${ }^{111}$ using the Heisenberg Hamiltonian

$$
\mathcal{H}=-\sum_{i \mu, j \nu} J_{i \mu, j \nu} \mathbf{S}_{i \mu} \cdot \mathbf{S}_{j \nu}-\sum_{i \mu} \epsilon_{\mu} H_{\mu} S_{i \mu}^{z}
$$

satisfactorily describes the experimental dispersion relation. The dominant exchange constants for $\mathrm{Cr}_{2} \mathrm{O}_{3}$ are $J=-7.5$ and $-3.3 \mathrm{meV}$ between spin pairs $A$ and $B$, respectively ${ }^{107}$ (refer to Fig. 3), while for $\alpha-\mathrm{Fe}_{2} \mathrm{O}_{3}$ spin pairs $C$ and $D$ interact most strongly with exchange constants $J=-2.6$ and $-2.0 \mathrm{meV}$, respectively. ${ }^{108}$ In $\mathrm{V}_{2} \mathrm{O}_{3}$, the corundum sym-

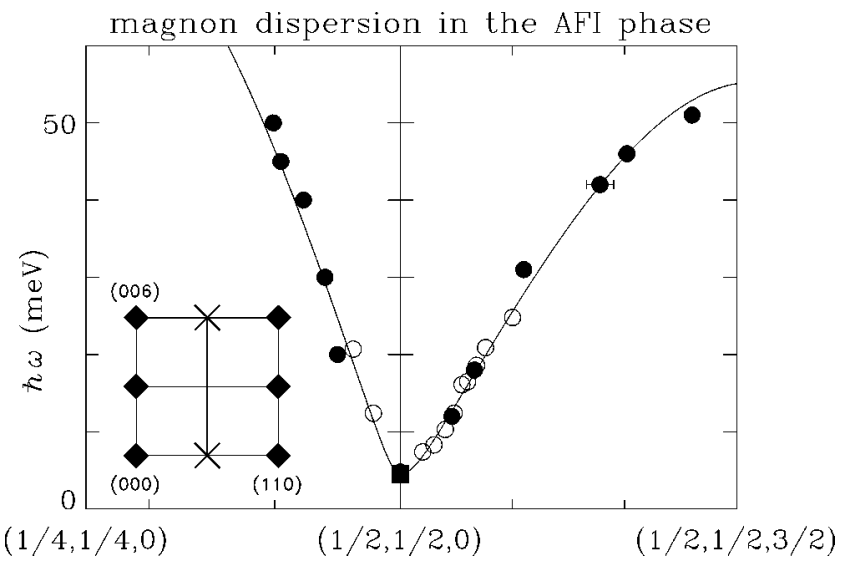

FIG. 18. Spin wave dispersion near the antiferromagnetic zone center. Solid circles represent the current measurements at $11 \mathrm{~K}$ for $\mathrm{V}_{1.944} \mathrm{Cr}_{0.056} \mathrm{O}_{3}$, the square is from Ref. 32. For comparison, data from Ref. 31 for pure $\mathrm{V}_{2} \mathrm{O}_{3}$ at $142 \mathrm{~K}$ are represented by open circles. With these limited data, individual exchange constants cannot be derived reliably. Only a specific linear combination as described in the text can be determined. Inset: the reciprocal-lattice plane for the measurement. The diamonds denote nuclear Bragg points, and crosses denote magnetic Bragg points.

metry is broken ${ }^{112,23}$ at the AFI transition. Consequently there are seven different exchange constants between these spin pairs [refer to Fig. 3(b)].

Dispersion relations for spin waves in the AFI phase of $\mathrm{V}_{2} \mathrm{O}_{3}$ are derived in Appendix A following the procedure described by Sáenz. ${ }^{113}$ The limited data in Fig. 18 do not allow reliable determination of individual exchange constants. Along the $(h h 0)$ direction the acoustic spin wave has a simple dispersion relation

$$
\begin{aligned}
{[\hbar \omega(\boldsymbol{\kappa})]^{2}=} & {\left[H_{0}-4 S\left(J_{\beta}+J_{\delta}+2 J_{\zeta}\right)\right]^{2} } \\
& -\left[4 S\left(J_{\beta}+J_{\delta}+2 J_{\zeta}\right)\right]^{2} \cos ^{2}(2 \pi h) .
\end{aligned}
$$

In combination with the previously measured value of the ordered moment $\left(g S=1.2^{24}\right)$, our measurements of this dispersion relation permit the local anisotropy field, $H_{0}$ $=0.13(2) \mathrm{meV}$, and $J_{\beta}+J_{\delta}+2 J_{\zeta}=33(2) \mathrm{meV}$ to be determined with confidence. ${ }^{114}$ To determine the individual exchange constants, experiments which will measure spin waves in the AFI across the whole Brillouin zone are now underway.

\section{THE INSULATING SPIN LIQUID}

The solid circles in Fig. 19 represent constant energy scans along $(10 l)$ at $205 \mathrm{~K}$ in the PI phase of $\mathrm{V}_{1.944} \mathrm{Cr}_{0.056} \mathrm{O}_{3}$. The peak profile is not sensitive to energy transfer for $1.6 \mathrm{meV} \leqslant \hbar \omega \leqslant 18 \mathrm{meV}$. The peaks are very broad and the half-width at half-maximum (HWHM) corresponds to a correlation length $\xi_{c} \sim 1.5 \AA$ in the c direction. Scans in the basal plane (refer to Fig. 20) also yielded broad $\hbar \omega$-insensitive peaks, corresponding to $\xi_{a} \sim 2.0 \AA$ in the $\mathbf{a}$ direction. These correlation lengths extend only to the nearest neighbors in the respective directions and they are even shorter than in the PM phase at comparable temperatures, as can be ascertained by comparing Fig. 12 or Fig. 15 to Fig. 19. This is a surprising result because the electron-hole pair 


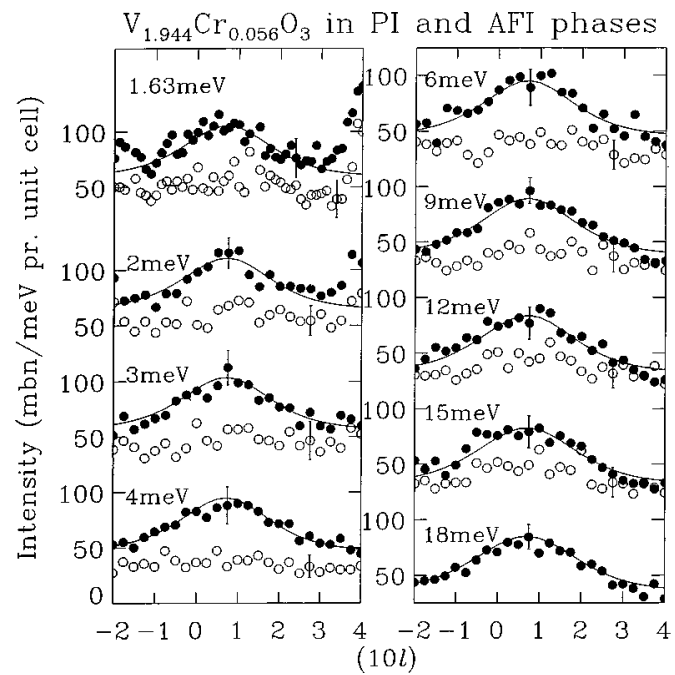

FIG. 19. Constant energy scans along the $\mathbf{c}$ direction in the PI phase at $205 \mathrm{~K}$ (solid circles) and in the AFI phase (open circles) for $\left(\mathrm{V}_{1-x} \mathrm{Cr}_{x}\right)_{2} \mathrm{O}_{3} \quad(x=0.028)$. Measurements in the AFI phase were conducted at $170 \mathrm{~K}$ for $\hbar \omega=6-12 \mathrm{meV}$ and at $160 \mathrm{~K}$ for other energies. Extra intensity near the nuclear Bragg points $(10 \overline{2})$ and (104) for $\hbar \omega \leqslant 2 \mathrm{meV}$ is probably due to phonons. Statistical uncertainty in intensity is indicated by the vertical bars. The spectrometer configurations at BT2 were $E_{i}=12.7 \mathrm{meV}$ with horizontal collimations $60^{\prime}-40^{\prime}-40^{\prime}-60^{\prime}$ for $\hbar \omega=1.63 \mathrm{meV}, \quad E_{f}$ $=13.7 \mathrm{meV}$ with $60^{\prime}-40^{\prime}-40^{\prime}-60^{\prime}$ for $\hbar \omega=2-15 \mathrm{meV}$, and $E_{i}$ $=35 \mathrm{meV}$ with $60^{\prime}-40^{\prime}-80^{\prime}-100^{\prime}$ for $\hbar \omega=18 \mathrm{meV}$.

damping mechanism which exists in the metal is inhibited by the charge excitation gap of the insulator.

The effect of temperature on the short-range magnetic correlations in the PI phase is shown in Fig. 21. Data for two values of energy transfer are shown. Clearly, q-dependent magnetic scattering is suppressed at high temperatures. Even so, there is no discernible narrowing of the peak, even as the AFI transition temperature, $T_{N}=180 \mathrm{~K}$, is approached. Thus, $T_{N}$ does not appear to be the critical temperature for spin fluctuations in the PI. As for stoichiometric $\mathrm{V}_{2} \mathrm{O}_{3}$ (refer to Fig. 15), the characteristic wave vector for magnetic correlations of $\mathrm{Cr}$-doped $\mathrm{V}_{2} \mathrm{O}_{3}$ moves to another part of reciprocal space in the AFI phase ${ }^{37}$ leaving a flat background as shown by open circles in Fig. 19. Therefore, the phase transition to the AFI is not a conventional antiferromagnetic transition, for which the correlation length diverges at $T_{N}$ and magnetic Bragg peaks emerge from peaks in the paramagnetic response function. Instead, the spin Hamiltonian is altered by some underlying processes.

Not only is the location in reciprocal space of the paramagnetic neutron scattering in the PI phase unusual, but also is the $\mathbf{q}$ and $\hbar \omega$ dependence of scattering about this location. For conventional insulating antiferromagnets, when the correlation length is limited to the nearest-neighbor spin spacing by thermal fluctuations at $T \gg J / k_{B}$, there is very little spectral weight in the modulated part of the dynamic correlation function. In the PI phase of $\mathrm{V}_{1.944} \mathrm{Cr}_{0.056} \mathrm{O}_{3}$, however, constant energy cuts through $\mathcal{S}(\mathbf{q}, \omega)$ show that large spectral weight $\left(>1.4 \mu_{B}\right.$ per $\mathrm{V}$ ion, see below) is associated with a ridge along $\hbar \omega$ which forms a very broad peak in $\mathbf{q}$ space. These experimental results suggest that the spin-spin interaction strength is not dwarfed by the thermal energy and that a
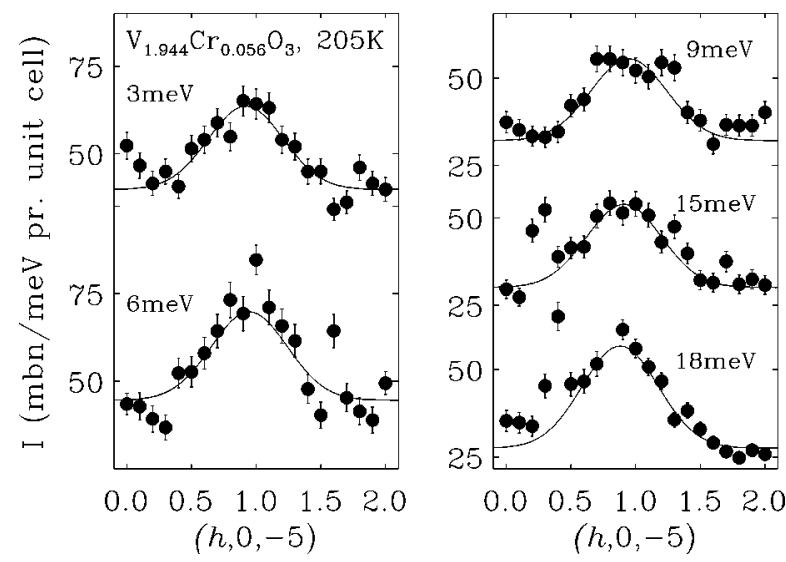

FIG. 20. Constant energy scans along the $\mathbf{a}^{*}$ direction in the PI phase at $205 \mathrm{~K}$ for $\left(\mathrm{V}_{1-x} \mathrm{Cr}_{x}\right)_{2} \mathrm{O}_{3} \quad(x=0.028)$. There is no appreciable energy dependence of the peak width. The spectrometer configurations at BT2 were $E_{f}=13.7 \mathrm{meV}$ with horizontal collimations $60^{\prime}-40^{\prime}-40^{\prime}-60^{\prime}$ for $\hbar \omega=3-15 \mathrm{meV}$ and $E_{i}=35 \mathrm{meV}$ with $60^{\prime}-40^{\prime}-80^{\prime}-100^{\prime}$ for $\hbar \omega=18 \mathrm{meV}$.

mechanism other than thermal fluctuations therefore must be responsible for limiting magnetic correlations to nearest neighbors.

One possible mechanism is geometric frustration where conflicting magnetic interactions prevent long-range order at $T \sim J / k_{B}$. Solid-state chemistry, however, does not support this explanation because the dominant exchange interactions in Heisenberg models for corundum antiferromagnets in general are not frustrated. ${ }^{115,116}$ Disorder introduced by $\mathrm{Cr}$ doping is also unlikely to be important, since for the same sample at low temperatures, spin waves in the AFI phase are resolution-limited (refer to Sec. V and Fig. 3 in Ref. 37). A very promising explanation, based on the electronic state of
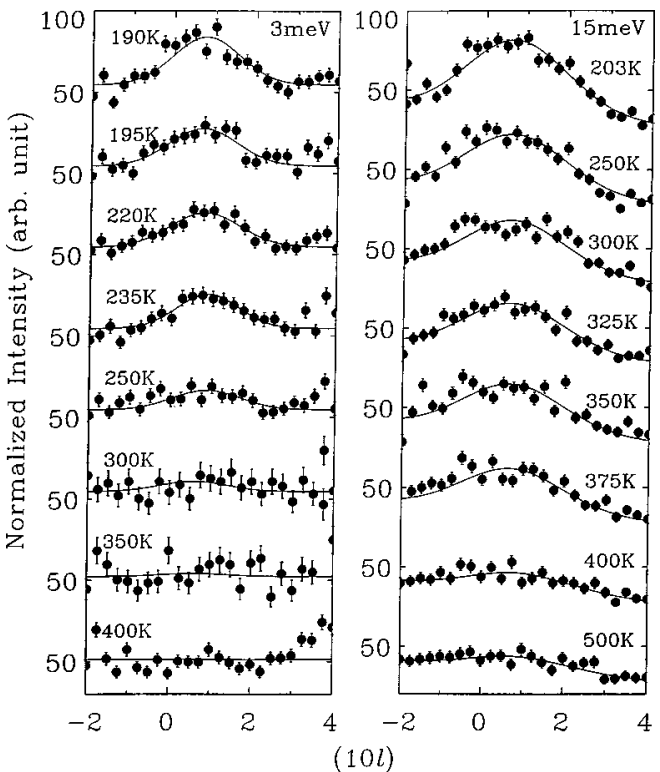

FIG. 21. Temperature dependence of constant energy scans in the PI phase of $\mathrm{V}_{1.944} \mathrm{Cr}_{0.056} \mathrm{O}_{3}$. The left panel is for $\hbar \omega=3 \mathrm{meV}$ and the right panel is for $\hbar \omega=15 \mathrm{meV}$. The spectrometer configurations at BT2 were $E_{f}=13.7 \mathrm{meV}$ with horizontal collimations $60^{\prime}-40^{\prime}-40^{\prime}-60^{\prime}$. The solid line is a fit to the product of the form factor $|f(\mathbf{q})|^{2}$ and a Gaussian function. 
$\mathrm{V}_{2} \mathrm{O}_{3}$ in the single ion limit, was recently proposed by Rice. ${ }^{117,37}$ Each magnetically active electron on a vanadium ion can exist in one of the two doubly degenerate $3 d$ orbitals. ${ }^{40}$ The orbital degeneracy may be the source of a large orbital contribution to the nuclear relaxation. ${ }^{118}$ Depending on the relative occupation of these two degenerate orbitals on neighboring sites, the exchange interaction between spins on these sites can have different signs. ${ }^{119}$ In the PI phase, the orbital occupation is fluctuating and disordered, resulting in a spin Hamiltonian with fluctuating exchange interactions of different signs which induce local antiferromagnetic correlations while dephasing longer-range correlations. We will return to this idea in Sec. VII.

\section{A. Analysis of spatial correlations}

A consequence of the $\hbar \omega$-insensitive peak width in the PI phase (Figs. 19 and 20) is that constant energy scans directly probe the $\mathbf{q}$ dependence of the instantaneous spin correlation function. Because of the short correlation lengths, the spatial dependence of the magnetic correlations can be modeled by a finite spin cluster which includes the four types of nearneighbor spin pairs: $A, B, C$, and $D$ [refer to Fig. 3(a)]. In other words, the summation in the structure factor

$$
\mathcal{S}(\mathbf{q}) \equiv \frac{\left(g \mu_{B}\right)^{2} n}{3}\left(\left\langle S_{\mathbf{0}}\right\rangle^{2}+\sum_{\mathbf{R}^{\prime}} \cos \left(\mathbf{q} \cdot \mathbf{R}^{\prime}\right)\left\langle\mathbf{S}_{\mathbf{0}} \cdot \mathbf{S}_{\mathbf{R}^{\prime}}\right\rangle\right)
$$

need only include one $A$-type spin pair, three $B$-type spin pairs, three $C$-type spin pairs, and six $D$-type spin pairs for any one spin, $\mathbf{S}_{\mathbf{0}}$, of the $n=12$ spins in a hexagonal unit cell.

In Fig. 22, an extended scan along the $\mathbf{c}$ axis, covering more than three Brillouin zones, and scans in the basal plane intersecting the peaks near the $(101)$ and $(10 \overline{5})$ are shown for $\mathrm{V}_{1.944} \mathrm{Cr}_{0.056} \mathrm{O}_{3}$ at $205 \mathrm{~K}$ in the PI phase. The background measured at $160 \mathrm{~K}$ in the AFI phase is also shown. A good approximation to the magnetic intensity at $205 \mathrm{~K}$ is obtained by subtracting the $160 \mathrm{~K}$ background and such difference data are shown in Fig. 23. The dotted line indicates the magnetic form factor for the $V^{3+}$ ion, ${ }^{55}|f(\mathbf{q})|^{2}$. Extra intensity at large $|\mathbf{q}|$ is attributed to incomplete subtraction of phonon neutron scattering. A simultaneous fit of $|f(\mathbf{q})|^{2} \mathcal{S}(\mathbf{q})$ to the magnetic intensity from these $3 \mathrm{meV}$ scans is shown as solid lines in Fig. 23. As can be seen in the figure, this spin cluster model is consistent with the data over the large part of $\mathbf{q}$ space where the magnetic form factor is appreciable.

The near-neighbor spin correlations derived from the best fit $\left(\chi^{2}=1.9\right)$ are

$$
\begin{gathered}
\left\langle\mathbf{S}_{\mathbf{0}} \cdot \mathbf{S}_{[0,0,1 / 6+\delta]}\right\rangle^{A} /\langle S\rangle^{2}=0.08(9), \\
\left\langle\mathbf{S}_{\mathbf{0}} \cdot \mathbf{S}_{[1 / 3,2 / 3, \bar{\delta}]}\right\rangle^{B} /\langle S\rangle^{2}=-0.10(4), \\
\left\langle\mathbf{S}_{\mathbf{0}} \cdot \mathbf{S}_{[2 / 3,1 / 3, \delta-1 / 6]}\right\rangle^{C} /\langle S\rangle^{2}=0.04(4), \\
\left\langle\mathbf{S}_{\mathbf{0}} \cdot \mathbf{S}_{[2 / 3,1 / 3,1 / 6]}\right\rangle^{D} /\langle S\rangle^{2}=-0.06(2),
\end{gathered}
$$

where $\delta=0.026$ and the superscripts $A-D$ refer to spin pairs in Fig. 3(a). Now, it is likely that warming yields an additional nonmagnetic background, most probably due to phonon and multiphonon scattering responsible for the upturn at

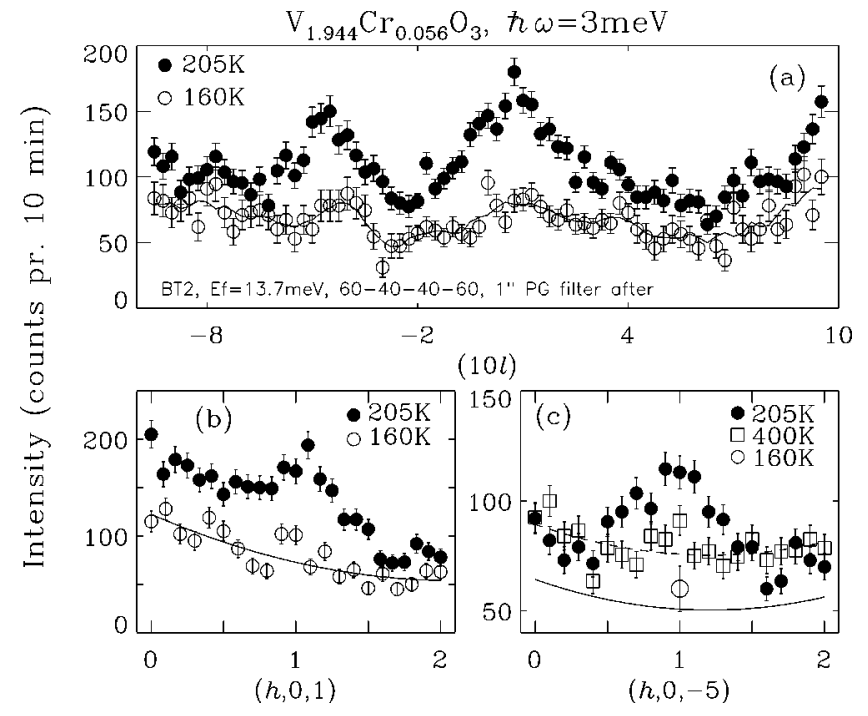

FIG. 22. Constant $\hbar \omega=3 \mathrm{meV}$ scans (a) along (10l), (b) along $(h, 0,1)$, and (c) along $(h, 0, \overline{5})$ at $205 \mathrm{~K}$ in the PI phase (solid circles) and at $160 \mathrm{~K}$ in the AFI phase (open circles). Squares in (c) represent data at $400 \mathrm{~K} .(1,0, \overline{8}),(1,0, \overline{2}),(1,0,4)$, and $(1,0,10)$ are nuclear Bragg points. The experimental configuration is indicated in (a).

high $|l|$ in Fig. 22(a). We account for this rising background by incorporating an additive constant in our fits, as we have done previously. ${ }^{35,37}$ The best least-squared fit $\left(\chi^{2}=1.2\right)$ is obtained with magnetic parameters 35,37

$$
\begin{gathered}
\left\langle\mathbf{S}_{\mathbf{0}} \cdot \mathbf{S}_{[0,0,1 / 6+\delta]}\right\rangle^{A} /\langle S\rangle^{2}=0.6(3), \\
\left\langle\mathbf{S}_{\mathbf{0}} \cdot \mathbf{S}_{[1 / 3,2 / 3, \bar{\delta}]}\right\rangle^{B} /\langle S\rangle^{2}=-0.19(8),
\end{gathered}
$$

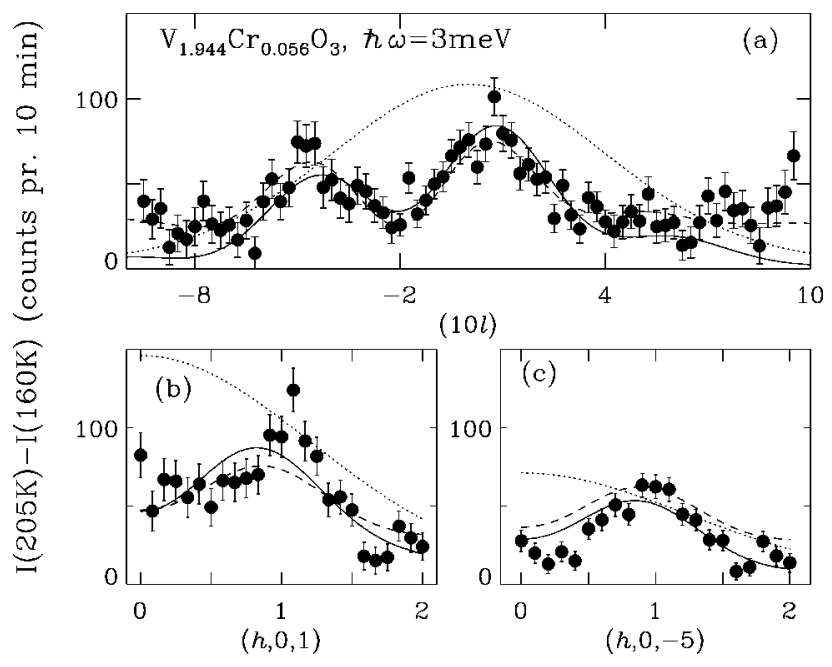

FIG. 23. Magnetic signal at $205 \mathrm{~K}$ in the PI phase, after subtracting the smoothed $160 \mathrm{~K}$ intensity (solid line in Fig. 22) as a measure of nonmagnetic backround. The background for (c) was determined from the $400 \mathrm{~K}$ and $160 \mathrm{~K}$ data. The dotted line represents the magnetic form factor $|f(\mathbf{q})|^{2}$. Extra intensity at large $|\mathbf{q}|$ is probably due to phonon scattering. The solid line represents $|f(\mathbf{q})|^{2} \mathcal{S}(\mathbf{q})$ calculated with the parameters of Eq. (26) while the dashed line is a constant plus this quantity calculated with the parameters of Eq. (27). 


$$
\begin{aligned}
& \left\langle\mathbf{S}_{\mathbf{0}} \cdot \mathbf{S}_{[2 / 3,1 / 3, \delta-1 / 6]}\right\rangle^{C} /\langle S\rangle^{2}=0.18(8), \\
& \left\langle\mathbf{S}_{\mathbf{0}} \cdot \mathbf{S}_{[2 / 3,1 / 3,1 / 6]}\right\rangle^{D} /\langle S\rangle^{2}=-0.09(3),
\end{aligned}
$$

and a constant background of 25 counts in $10 \mathrm{~min}$.

The physical picture which emerges from both fits is that in the PI phase, the nearest-neighbor spin pairs in the basal plane, which contains a puckered honeycomb lattice for $\mathrm{V}$, are more likely to be antiparallel to each other, while the nearest-neighbor spin pairs in the $\mathbf{c}$ direction are more likely to be parallel to each other. While the signs of correlations for different pairs are clear, the specific values of the spin pair correlations have large statistical error bars and possibly systematic errors associated with uncertainty in the background determination. If this dynamic short-range spin correlations were allowed to develop into a long-range order, the spin correlations would be

$$
\begin{gathered}
\left\langle\mathbf{S}_{\mathbf{0}} \cdot \mathbf{S}_{[0,0,1 / 6+\delta]}\right\rangle^{A} /\langle S\rangle^{2}=1, \\
\left\langle\mathbf{S}_{\mathbf{0}} \cdot \mathbf{S}_{[1 / 3,2 / 3, \bar{\delta}]}\right\rangle^{B} /\langle S\rangle^{2}=-1, \\
\left\langle\mathbf{S}_{\mathbf{0}} \cdot \mathbf{S}_{[2 / 3,1 / 3, \delta-1 / 6]}\right\rangle^{C} /\langle S\rangle^{2}=1, \\
\left\langle\mathbf{S}_{\mathbf{0}} \cdot \mathbf{S}_{[2 / 3,1 / 3,1 / 6]}\right\rangle^{D} /\langle S\rangle^{2}=-1 .
\end{gathered}
$$

This corresponds to the type-I collinear antiferromagnetic structure for corundum. ${ }^{115}$

In Fig. 24, the magnetic intensities of Fig. 19 from $\hbar \omega$ $=1.63 \mathrm{meV}$ to $18 \mathrm{meV}$ have been integrated, to yield the quasi-instantaneous correlation function

$$
\begin{aligned}
\hbar \int d \omega \mathcal{S}(\mathbf{q}, \omega) & \approx \frac{\left(g \mu_{B}\right)^{2}}{3 N} \sum_{\mathbf{R R}^{\prime}} e^{i \mathbf{q} \cdot\left(\mathbf{R}^{\prime}-\mathbf{R}\right)}\left\langle\mathbf{S}_{\mathbf{R}}(\delta t) \cdot \mathbf{S}_{\mathbf{R}^{\prime}}(0)\right\rangle \\
& \approx \mathcal{S}(\mathbf{q}),
\end{aligned}
$$

where $|\delta t|<\hbar / 18 \mathrm{meV}=3.7 \times 10^{-14}$ sec. The solid line in the figure is $\mathcal{S}(\mathbf{q})$, calculated from Eqs. (25) and (26) using $\left(g \mu_{B}\right)^{2}\langle S\rangle^{2} / 3=\left(0.80 \mu_{B}\right)^{2}$. Thus, at $205 \mathrm{~K}$, the fluctuating moment involved in the short-range correlations, up to $\hbar \omega$ $=18 \mathrm{meV}$, is $1.4(2) \mu_{B}$ per $\mathrm{V}$. This is as large as the ordered moment of $1.2 \mu_{B}$ per $\mathrm{V}$ in the AFI phase.

\section{B. Energy dependence}

Figure 25(a) shows the energy dependence of $\chi^{\prime \prime}(\mathbf{Q}, \omega)$ at $\mathbf{Q}=(1,0,0.76)$ for $\mathrm{V}_{1.944} \mathrm{Cr}_{0.056} \mathrm{O}_{3}$ at $205 \mathrm{~K}$. The data were obtained from the peak intensities of the constant-energy scans in Fig. 19. There is a remarkable similarity between these data and the peak intensities for metallic pure $\mathrm{V}_{2} \mathrm{O}_{3}$ shown in Fig. 25(c) despite the difference in $S(\mathbf{q})$ for these two samples (see, e.g., Figs. 12 and 19).

The spectrum can be modeled by a Lorentzian

$$
\chi^{\prime \prime}(\mathbf{q}, \omega)=\frac{\chi(\mathbf{q})}{\Gamma_{\mathbf{q}}} \frac{\hbar \omega}{1+\left(\hbar \omega / \Gamma_{\mathbf{q}}\right)^{2}},
$$

where $\chi(\mathbf{q})$ is a generalized $\mathbf{q}$-dependent static susceptibility and $\Gamma_{\mathbf{q}}$ is the relaxation energy. The data in Fig. 25(a) only allow us to place a lower limit of $18 \mathrm{meV}$ on $\Gamma_{\mathbf{Q}}$ at $T$ $=205 \mathrm{~K}$. The initial slope

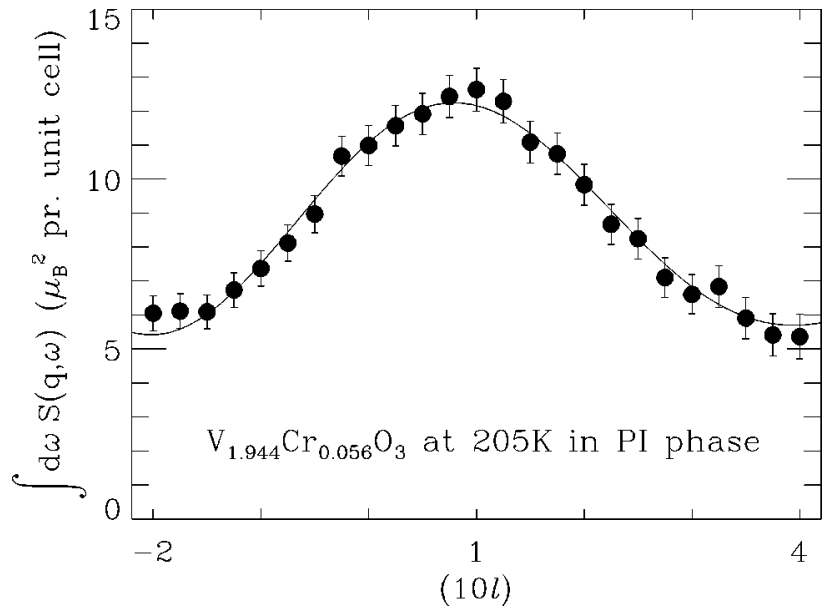

FIG. 24. Energy integrated intensity, up to $18 \mathrm{meV}$, as a function of $\mathbf{q}=(10 l)$ at $205 \mathrm{~K}$ in the PI phase for $\mathrm{V}_{1.944} \mathrm{Cr}_{0.056} \mathrm{O}_{3}$. The solid line is $12 \mathcal{S}(\mathbf{q})$ based on Eq. (25), the parameters of Eq. (26), and $g^{2} S^{2} / 3=0.80^{2}$.

$$
\Pi(\mathbf{Q}) \equiv \lim _{\omega \rightarrow 0} \chi^{\prime \prime}(\mathbf{Q}, \omega) / \hbar \omega=\chi(\mathbf{Q}) / \Gamma_{\mathbf{Q}}
$$

is, however, reliably determined to be $\Pi(\mathbf{Q})$ $=0.075(4) \mu_{B}^{2} / \mathrm{meV}^{2}$ per unit cell.

The energy dependence of the local magnetic susceptibility

$$
\chi^{\prime \prime}(\omega) \equiv \int d^{3} \mathbf{q} \chi^{\prime \prime}(\mathbf{q}, \omega) / \int d^{3} \mathbf{q}
$$

for the PI and PM are shown in Fig. 25(b) and 25(d). For the insulating sample these data were derived from the Gaussian fits shown in Figs. 19 and 20 while we used the fits shown in Fig. 15 for the metallic sample. Again we observe that for $T \approx 200 \mathrm{~K}$ the magnetic spectral weight for $\hbar \omega<25 \mathrm{meV}$ is substantially larger in the PI phase than in the PM phase.

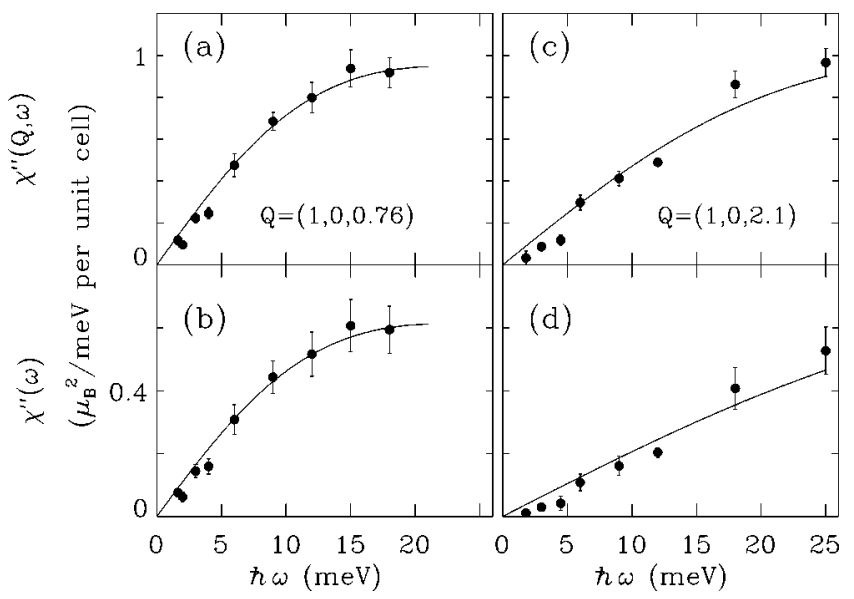

FIG. 25. (a) The dynamic susceptibility at $\mathbf{Q}$ specified in the figure, and (b) the local dynamic susceptibility as a function of energy for $\mathrm{V}_{1.944} \mathrm{Cr}_{0.056} \mathrm{O}_{3}$ at $205 \mathrm{~K}$ in the $\mathrm{PI}$ phase. The corresponding quantities for stoichiometric $\mathrm{V}_{2} \mathrm{O}_{3}$ at $200 \mathrm{~K}$ in the PM phase are shown in (c) and (d). 


\section{Comparison to bulk susceptibility data}

As a check on the reliability of our normalization procedures, we compare the normalized dynamic susceptibilities at $T=205 \mathrm{~K}$ discussed above to the conventional bulk susceptibility, $\quad \chi \equiv \chi^{\prime}(\mathbf{0}, 0)=\chi(\mathbf{q}=\mathbf{0})+\chi^{\prime}(\mathbf{0}, \infty)$ for $\mathrm{V}_{1.944} \mathrm{Cr}_{0.056} \mathrm{O}_{3}$.

From Eq. (29) and the Kramers-Kronig relation, as long as we are in the classic regime, $\Gamma_{\mathbf{q}}<k_{B} T$,

$$
\mathcal{S}(\mathbf{q}) \equiv \hbar \int d \omega \chi^{\prime \prime}(\mathbf{q}, \omega)\langle n(\omega)+1\rangle \approx k_{B} T \chi(\mathbf{q})
$$

Therefore, at a given temperature,

$$
\chi(\mathbf{0}) \approx \frac{\mathcal{S}(\mathbf{0})}{\mathcal{S}(\mathbf{Q})} \chi(\mathbf{Q})=\frac{\mathcal{S}(\mathbf{0})}{\mathcal{S}(\mathbf{Q})} \Gamma_{\mathbf{Q}} \Pi(\mathbf{Q})
$$

Using parameters in Eqs. (26) or (27) for our finite cluster model, we find $S(\mathbf{Q}) / S(\mathbf{0}) \approx 3.0$ or 2.2 . Thus, assuming that $\Gamma_{\mathbf{Q}} \lesssim k_{B} T$, we can estimate the bulk susceptibility $\chi(\mathbf{0})$ from the parameters quoted in Sec. VIB. The result is $\chi \geqslant 0.5-0.7 \mu_{B}^{2} / \mathrm{meV}$ per unit cell $=(1.1-1.6)$ $\times 10^{-3} \mathrm{emu} / \mathrm{mol} \mathrm{V}$ for $\mathrm{V}_{1.944} \mathrm{Cr}_{0.056} \mathrm{O}_{3}$ at $T=205 \mathrm{~K}$. This number should be compared to the measured bulk susceptibility which is $3.6 \times 10^{-3} \mathrm{emu} / \mathrm{mol} \mathrm{V}$ at $205 \mathrm{~K}^{83}$

\section{Temperature dependence}

The temperature dependence of $\chi^{\prime \prime}(\mathbf{Q}, \omega) / \hbar \omega$ is shown in Fig. 26 for $\mathrm{V}_{1.944} \mathrm{Cr}_{0.056} \mathrm{O}_{3}$. The $3 \mathrm{meV}$ and $15 \mathrm{meV}$ data are from Fig. 21 and the $9 \mathrm{meV}$ data from Fig. 4(c) of Ref. 37. The values of $\chi^{\prime \prime}(\mathbf{Q}, \omega) / \hbar \omega$ from different $\hbar \omega$ $=3-15 \mathrm{meV}$ are close to each other down to $T_{N}=180 \mathrm{~K}$. This indicates that $\chi^{\prime \prime}(\mathbf{Q}, \omega) \propto \hbar \omega$ in this energy range and that $\Gamma_{\mathbf{Q}}$ therefore exceeds $15 \mathrm{meV}$ even close to the AFI transition [refer to Eq. (29)]. This also means that the ratios plotted are a measure of $\Pi(\mathbf{Q})$ [Eq. (30)].

In a second-order antiferromagnetic transition, $\chi(\mathbf{q}) \propto(T$ $\left.+T_{N}^{0}\right)^{-1}$ and $\Gamma_{\mathbf{Q}} \propto T-T_{N}^{0}$ in the Gaussian approximation, ${ }^{120}$ therefore, $\Pi(\mathbf{Q}) \propto\left(T^{2}-T_{N}^{02}\right)^{-1}$ scales as $T^{-2}$ for $T \gg T_{N}^{0}$. As expected, experimental data for metallic $\mathrm{V}_{1.973} \mathrm{O}_{3}$ follow this relation for $T \gg T_{N}=9 \mathrm{~K}$ (refer to the diamonds in Fig. 26). Interestingly, the data for the temperature dependence of $\Pi(\mathbf{Q})$ in $\mathrm{V}_{1.944} \mathrm{Cr}_{0.056} \mathrm{O}_{3}$, are proportional to $T^{-2}$ (refer to the solid line) all the way down to $T_{N}=180 \mathrm{~K}$. Therefore, the spin system in the PI phase at temperatures even slightly above the PI-AFI phase transition appears to be far away from the criticality associated with the real $T_{N}$. On the other hand, $T_{N}^{0}$ is remarkably close to the Néel temperature of the metallic AFM found for hole-doped samples. In other words, the PI fluctuations are those which one would associate with nearly the same quantum critical point as found for the PM and AFM phases.

\section{DISCUSSION AND CONCLUSIONS}

In the preceding sections, the magnetic properties of $\mathrm{V}_{2} \mathrm{O}_{3}$ in each of its phases were described separately. Interest in this material, however, is driven by attempts to understand the Mott-Hubbard transition. Here, we will discuss the implications of our magnetic neutron scattering experiments

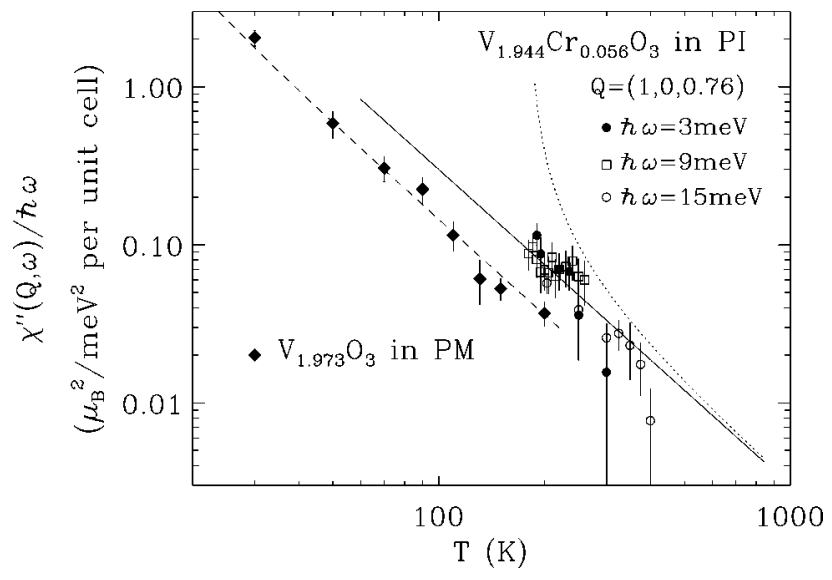

FIG. 26. Temperature variation of $\chi^{\prime \prime}(\mathbf{Q}, \omega) / \hbar \omega$ at $\mathbf{Q}$ $=(1,0,0.76)$ for $\mathrm{V}_{1.944} \mathrm{Cr}_{0.056} \mathrm{O}_{3}$ in the PI phase, with $\hbar \omega$ used in the measurement indicated in the figure. The corresponding quantity at the low-energy limit, $\chi_{\mathrm{Q}} / \gamma_{A} \kappa^{2}$, for $\mathrm{V}_{1.973} \mathrm{O}_{3}$ in PM (diamonds) is included for reference. The solid line is proportional to $T^{-2}$, the dotted line is proportional to $\left[T^{2}-(180 \mathrm{~K})^{2}\right]^{-1}$, and the dashed line is proportional to $\left[T^{2}-(9 \mathrm{~K})^{2}\right]^{-1}$.

for the interpretation of the transitions between the PI, AFI, PM, and AFM phases.

The discovery of antiferromagnetic order in metallic $\mathrm{V}_{2} \mathrm{O}_{3}$ through the ${ }^{57} \mathrm{Fe}$ Mössbauer effect ${ }^{63}$ prompted much theoretical work on antiferromagnetism in strongly correlated Fermi liquids. Phase diagrams including a metallic antiferromagnetic phase for the Mott system were subsequently published by several authors. ${ }^{28-30,12}$ However, for many of these studies, a Heisenberg antiferromagnetic state like that in the insulator was put into the metallic state by hand and local moment spin dynamics in this phase was implicit. Even though the effective mass of electrons in metallic $\mathrm{V}_{2} \mathrm{O}_{3}$ is strongly enhanced, i.e., the Fermi liquid is nearly localized near the metal-insulator transition, we showed in the preceding sections that the antiferromagnetism in metallic $\mathrm{V}_{2} \mathrm{O}_{3}$ is completely different from the antiferromagnetism in the AFI. While Heisenberg models of exchange coupled unpaired spins located at $\mathrm{V}$ ions can account for magnetic phenomena in the AFI phase, antiferromagnetism in the metal is controlled fundamentally by the Fermi sea. ${ }^{33,36,70}$ Specifically the small-moment static SDW order results from a nesting or near-nesting Fermi surface, and the broad bandwidth spin excitation spectrum is reminiscent of an electron-hole pair continuum. In short, our experiments show that the metalinsulator transition separates two qualitatively different antiferromagnetic states, with localized moments in the AFI and itinerant moments in the AFM phase.

With the large number of spin fluctuation modes in the Stoner continuum, spin fluctuations in an itinerant antiferromagnet have a stronger renormalizing effect on equilibrium magnetic properties than spin waves have in an insulating antiferromagnet. This makes magnetism at finite temperatures for itinerant magnets much more complex than for localized moment magnets. Near the quantum critical point in the small moment limit, scaling relations can be derived based on general physical arguments. ${ }^{84-87}$ The scaling functions and relations between nonuniversal constants can be further calculated by the SCR theory. ${ }^{18,95,17,105} \mathrm{We}$ found remarkable quantitative consistency between experimental re- 
sults for metallic $\mathrm{V}_{2-y} \mathrm{O}_{3}$ and the theory.

The source of an itinerant antiferromagnetic instability, which is characterized by the singularity of generalized magnetic susceptibility at finite $\mathbf{q}$, could be a divergent Lindhard function due to nesting Fermi surface, ${ }^{67,69}$ a nearly nesting Fermi surface in combination with the Coulomb interactions, ${ }^{69,121}$ or solely the strong Coulomb interactions. The last possibility is still under theoretic investigations. ${ }^{122,123}$ The nesting scenario has been extremely successful in describing the classic itinerant antiferromagnet, $\mathrm{Cr} .{ }^{79} \mathrm{We}$ find it also provides a comprehensive framework for metallic $\mathrm{V}_{2} \mathrm{O}_{3} \cdot{ }^{33,36}$ In contrast to $\mathrm{Cr}$, however, only a small area of the Fermi surface is involved in the SDW, ${ }^{33}$ and strong correlations in metallic $\mathrm{V}_{2} \mathrm{O}_{3}$ require only near nesting of the Fermi surface for the long-range antiferromagnetic state to occur.

Three kinds of metal-insulator transitions occur in the $\mathrm{V}_{2} \mathrm{O}_{3}$ system: the PM-PI transition, the PM-AFI transition, and the $T \rightarrow 0$ AFM-AFI transition. Whether they should be called Mott transitions has been controversial and, to some extent, is confused by the evolving meaning of the Mott transition. While previously noticed by others, Mott ${ }^{124}$ was responsible for bringing attention to the possibility of localization of electrons in a narrow band through electronic correlations, which is neglected in conventional band theories of solids. Mott argued for a first-order metal-insulator transition when the electron density is reduced below some critical value. Not much detail about the electronic processes taking place at the transition were known at that time, and since the "exceptional" insulators such as $\mathrm{NiO},{ }^{125}$ which motivated Mott's research and are today called Mott and chargetransfer insulators, were known experimentally to be antiferromagnets, it was unclear whether there was any new physics apart from magnetism. Specifically, if these insulators are always antiferromagnetic, a doubling of the unit cell will open a gap at the Fermi level as shown by Slater. ${ }^{27}$ However, Hubbard ${ }^{3}$ demonstrated in 1964 that a metal-insulator transition can be produced by strong Coulomb correlations without antiferromagnetic ordering. Brinkman and Rice ${ }^{6}$ in 1970 outlined a distinct type of Fermi liquid on the metallic side of the Mott-Hubbard metal-insulator transition. Strong electron correlations in the PM phase of $\mathrm{V}_{2} \mathrm{O}_{3}$ were revealed through its Brinkman-Rice-like behavior ${ }^{126}$ and the PM-PI transition was discovered as a possible experimental realization of the Mott-Hubbard transition by McWhan, Rice, and Remeika. ${ }^{127}$ This probably represents the first explicitly identified MottHubbard transition in a real material.

The difference between the paramagnetic metal and the paramagnetic insulator in $\left(\mathrm{V}_{1-x} \mathrm{Cr}_{x}\right)_{2} \mathrm{O}_{3}$ disappears at the critical point near $400 \mathrm{~K}$ (or the critical line in the composition-P-T phase space ${ }^{128}$ ). A continuous crossover between paramagnetic spin fluctuations in the metal and the insulator thus is expected on a path above the critical point. By inspecting the evolution of magnetic correlations with temperature and doping in the metal (refer to Figs. 10, 12, and 15) and in the insulator (refer to Figs. 19 and 21), such a magnetic crossover from metal to insulator can be conceived as follows: The pair of incommensurate peaks in the metal broaden in $\mathbf{q}$ upon approaching the critical point until they finally merge into the single broad peak which we observe in the PI. The blurring of the incommensurate peaks, which is a measure of Fermi surface dimension on the nesting parts, parallels the losing of metallic coherence among electrons.

Although Hubbard as well as Brinkman and Rice have shown that a Mott transition does not require an antiferromagnetic transition, antiferromagnetic order does promote the insulating state. This is apparent because of the enlarged insulating phase space associated with the AFI in various studies based on the one-band Hubbard model. ${ }^{28,29,12}$ The resemblance of the theoretical phase diagrams to the experimental phase diagram of $\mathrm{V}_{2} \mathrm{O}_{3}$ gives merit to Slater's idea of an insulator induced by antiferromagnetism.

However, an important difference exists between these one-band phase diagrams and the $\mathrm{V}_{2} \mathrm{O}_{3}$ phase diagram. The magnetic transition in the theories is predicted to be of second order while the experimental AFI transition in $\mathrm{V}_{2} \mathrm{O}_{3}$ is first order [refer, e.g., to Fig. 8(a)]. This difference is not so much trivial as merely a magnetostriction effect. Beneath it is a fundamental difference in temperature dependence of spatial spin correlations. ${ }^{37}$ It is well known ${ }^{129}$ that a oneband Hubbard model reduces to a Heisenberg model with exchange $J \sim t^{2} / U$ in the insulating limit $U / t \gg 1$. For temperatures lower than the insulating gap $\sim U / k_{B}$, the lowenergy physics is contained in the Heisenberg model with a second-order antiferromagnetic transition at $T_{N} \sim J / k_{B}$. The characteristic magnetic wave vector remains the same for $T$ $>T_{N}$ and $T<T_{N}$. In $\mathrm{V}_{2} \mathrm{O}_{3}$, dynamic spin fluctuations in the corundum PM and PI phases peak at a magnetic wave vector along the $\mathbf{c}^{*}$ axis. These magnetic correlations abruptly vanish and are replaced by $(1 / 2,1 / 2,0)$-type long range order and the associated spin waves below the first-order AFI transition (see Ref. 37 for details; see also Figs. 15 and 19 in this paper). This switching between spin correlations with different magnetic wave vectors indicates that the antiferromagnetic transition is not a common order-disorder phase transition, as is found, for example, in the parent compounds of high- $T_{C}$ superconductors, but that instead the spin Hamiltonian is somehow modified at the first-order transition to the AFI phase. These results find no comprehensive explanation in one-band Hubbard models.

We mentioned in Sec. VI that the electronic state of $\mathrm{V}_{2} \mathrm{O}_{3}$ in the single-ion limit can be approximated by two degenerate $d$ orbitals at each $\mathrm{V}$ ion site which are filled by a single electron. New physics in Hubbard models with degenerate bands was discussed by several authors more than twenty years ago. ${ }^{130-132,119}$ It gains renewed interest in light of recent experimental progress. ${ }^{117,133,134}$ Depending on which one of the degenerate orbitals is occupied at a given site and at its neighboring sites, the exchange interaction between the nearest-neighbor spins is different and can even change sign. Thus, besides the spin degrees of freedom, a new orbital degree of freedom enters the low-energy physics. The spin and orbital degrees of freedom are strongly coupled, and the effective spin Hamiltonian depends sensitively on orders in the orbital degrees of freedom. ${ }^{117,119}$ Switching between spin correlations with different magnetic wave vectors was also recently observed in manganite at an orbital/charge ordering transition. ${ }^{135}$

The anomalously short-range spin fluctuations in the PI and the abrupt switching of magnetic correlations at the AFI transition can be understood, as first pointed out by Rice, ${ }^{117}$ if the primary order parameter for the AFI transition involves 
orbital degrees of freedom. At low temperatures, order in orbital occupations breaks the threefold corundum symmetry. Pairs with different orbitals at neighboring sites produce different bond strengths and different exchange interactions, resulting in the structural distortion and the antiferromagnetic structure in Fig. 3(b) which has two antiferromagnetic bonds and one ferromagnetic bond in the basal plane. ${ }^{119}$ In the PI phase the orbital occupation fluctuates between the two states of the doublet, thus restoring the corundum symmetry and producing a different spin Hamiltonian with random and fluctuating exchange interactions. With this kind of spin Hamiltonian, magnetic correlations necessarily are limited to the nearest neighbors. The fact that the magnetic transition is strongly first order indicates that the intrinsic Néel temperature, $T_{N}^{\mathrm{AFI}}$, of the spin Hamiltonian associated with the AFI phase is larger than the orbital ordering temperature $T_{O}$. Thus, when orbitals order and the spin Hamiltonian changes from the one associated with the PI phase, which has a $T_{N}^{\mathrm{PI}}<T_{O}$, to the one associated with the AFI phase, the magnetic moment reaches from zero directly to a value appropriate for $T=T_{O} \ll T_{N}^{\mathrm{AFI}}$.

In conclusion, we have shown that the metal-insulator transition fundamentally changes the nature of antiferromagnetism in the $\mathrm{V}_{2} \mathrm{O}_{3}$ system. Metallic $\mathrm{V}_{2} \mathrm{O}_{3}$ is a prototypical strongly correlated small-moment itinerant antiferromagnet, involving a nesting instability on a small part of the Fermi surface. We have also presented strong indications that orbital degeneracy plays a crucial role in the magnetism and phase transitions of this material. Specifically, it appears that these orbital degrees of freedom give rise to a novel fluctuating paramagnetic insulator, and a first-order transition to an antiferromagnetic phase with a spin structure which is unrelated to the paramagnetic short-range order. At the same time, the fluctuations in the paramagnetic insulator and paramagnetic metal are so similar that they suggest that the electron-hole pair excitations associated with Fermi surface nesting in the metal are transformed into orbital fluctuations in the insulator.

\section{ACKNOWLEDGMENTS}

We gratefully acknowledge discussions and communications with T. M. Rice, S. K. Sinha, Q. M. Si, G. Sawatzky, L. F. Mattheiss, G. Kotliar, C. Castellani, A. Fujimori, M. Takigawa, A. J. Millis, R. J. Birgeneau, G. Shirane, S. M. Shapiro, C. Varma, and T. Moriya. W. B. would like to thank R. W. Erwin, J. W. Lynn, D. A. Neumann, and J. J. Rush for their hospitality during experiments at NIST. W.B. and C.B. thank C. L. Chien and Q. Xiao for use of their laboratory for sample preparations. The work at JHU was supported by the NSF through Grant No. DMR-9453362, at BNL by DOE under Contract No. DE-AC02-98CH10886, at ORNL by DOE under Contract No. DE-AC05-96OR22464, at Purdue by MISCON Grant No. DE-FG02-90ER45427, and at the University of Chicago by the NSF through Grant No. DMR9507873.

\section{APPENDIX A: SPIN WAVES IN AFI}

Spin wave excitations from a collinear spin arrangement were studied by Sáenz. ${ }^{13}$ For a material with $n$ magnetic ions in a primitive magnetic unit cell, the positions of these ions are described by

$$
\mathbf{R}_{i \mu}=\mathbf{R}_{i}+\mathbf{r}_{\mu},
$$

where $\mathbf{R}_{i}$ is the location of the $i$ th unit cell $(i=1,2, \ldots, N)$, and $\mathbf{r}_{\mu}$ is the position of the $\mu$ th ion inside the cell $(\mu$ $=1,2, \ldots, n)$. The vector spin operator for the ion denoted by $\{i \mu\}$ is $\mathbf{S}_{i \mu}$ and the spin quantum number is $S_{\mu}$. Choosing $\hat{z}$ to be parallel to the collinear direction, the spin orientations are described by $\epsilon_{\mu}= \pm 1$ for up or down spins in a unit cell. A diagonal $n \times n$ matrix, E, with elements $\epsilon_{\mu} \delta_{\mu \nu}$ is introduced for later use.

The spin Hamiltonian is given by

$$
\mathcal{H}=-\sum_{i \mu, j \nu} J_{i \mu, j \nu} \mathbf{S}_{i \mu} \cdot \mathbf{S}_{j \nu}-\sum_{i \mu} \epsilon_{\mu} H_{\mu} S_{i \mu}^{z},
$$

where $H_{\mu}$ models the anisotropy field and the collinear component of the external field on the $\mu$ th ion, and the exchange interaction $J_{i \mu, j \nu}$ depends only on $\mu, \nu$ and the ionic separation $\mathbf{R}_{i \mu}-\mathbf{R}_{j \nu}$. As usual, $J_{i \mu, j \nu}=J_{j \nu, i \mu}$ and $J_{i \mu, i \mu}=0$.

The lattice transformation of the exchange constants is an $n \times n$ Hermitian matrix function and its elements are

$$
\mathrm{J}_{\mu \nu}(\boldsymbol{\kappa})=\sum_{j} J_{j \nu, 0 \mu} \exp \left[i \boldsymbol{\kappa} \cdot\left(\mathbf{R}_{j \nu}-\mathbf{R}_{0 \mu}\right)\right],
$$

and $\boldsymbol{\kappa}$ is defined within the first Brillouin zone. Introduce the matrix $\mathrm{L}(\boldsymbol{\kappa})$ :

$$
\begin{aligned}
& \mathrm{L}_{\mu \nu}(\boldsymbol{\kappa}) \triangleq 2\left(\epsilon_{\mu} \sum_{v} \mathrm{~J}_{\mu \nu}(0) \epsilon_{\nu} S_{v}+\frac{1}{2} H_{\mu}\right) \delta_{\mu \nu} \\
&-2 \epsilon_{\mu} \epsilon_{\nu}\left(S_{\mu} S_{\nu}\right)^{1 / 2} \mathrm{~J}_{\mu \nu}(\boldsymbol{\kappa})
\end{aligned}
$$

which is Hermitian $\mathrm{L}^{\dagger}(\boldsymbol{\kappa})=\mathrm{L}(\boldsymbol{\kappa})$ and $\mathrm{L}(-\boldsymbol{\kappa})=\mathrm{L}^{*}(\boldsymbol{\kappa})$. The $n$ eigenvalues, $\lambda_{\mu}(\boldsymbol{\kappa})$, of EL are related to the energies of the $n$ spin wave modes by

$$
\hbar \omega_{\mu}(\boldsymbol{\kappa})=\left|\lambda_{\mu}(\boldsymbol{\kappa})\right| .
$$

For a stable spin structure, the matrix $L(\boldsymbol{\kappa})$ is non-negative definite for every $\boldsymbol{\kappa}$, and $\lambda_{\mu}(\boldsymbol{\kappa})$ does not cross the zero axis for any branch of the dispersion relation, i.e., it is either non-negative or nonpositive throughout the Brillouin zone. The number of non-negative (nonpositive) branches of $\lambda_{\mu}(\boldsymbol{\kappa})$ equals the number of positive (negative) elements of $\epsilon_{\mu}$.

The spin Hamiltonian can now be rewritten in terms of spin wave modes,

$$
\mathcal{H}=-N\left(\sum_{j \nu} J_{0 \mu, j \nu} S_{\mu} S_{\nu}+\sum_{\mu} H_{\mu} S_{\mu}\right)+\hbar \sum_{\boldsymbol{\kappa}, \mu} \omega_{\mu}(\boldsymbol{\kappa}) n_{\mu}(\boldsymbol{\kappa})
$$

in the semiclassic limit, ${ }^{136}$ namely, $S_{\mu}-\epsilon_{\mu}\left\langle S_{i \mu}^{z}\right\rangle \ll 2 S_{\mu}$.

The primitive magnetic unit cell ${ }^{31}$ in the monoclinic AFI phase of $\mathrm{V}_{2} \mathrm{O}_{3}$ is shown in Fig. 3(b) together with the conventional hexagonal cell of $R \overline{3} c$ symmetry. Neglecting the small lattice distortion in the phase transition to the AFI, the monoclinic and hexagonal unit cells are related by the transformation 


$$
\left(\begin{array}{l}
\mathbf{a}_{m} \\
\mathbf{b}_{m} \\
\mathbf{c}_{m}
\end{array}\right)=\left(\begin{array}{rrr}
1 / 3 & -1 / 3 & 1 / 6 \\
1 / 3 & -1 / 3 & -1 / 3 \\
1 & 1 & 0
\end{array}\right)\left(\begin{array}{l}
\mathbf{a}_{h} \\
\mathbf{b}_{h} \\
\mathbf{c}_{h}
\end{array}\right)
$$

and

$$
\left(\begin{array}{c}
\mathbf{a}_{h} \\
\mathbf{b}_{h} \\
\mathbf{c}_{h}
\end{array}\right)=\left(\begin{array}{rrr}
1 & 1 / 2 & 1 / 2 \\
-1 & -1 / 2 & 1 / 2 \\
2 & -2 & 0
\end{array}\right)\left(\begin{array}{l}
\mathbf{a}_{m} \\
\mathbf{b}_{m} \\
\mathbf{c}_{m}
\end{array}\right)
$$

Denoting the above two matrices as $A$ and $B$, respectively, the corresponding transformation matrices for reciprocallattice primitive vectors are $\mathrm{B}^{T}$ and $\mathrm{A}^{T}$.

There are four magnetic $\mathrm{V}$ ions per monoclinic unit cell [refer to Fig. 3(b)] and they are assumed to have equivalent spins, $S_{\mu}=S$. With the designation of ions, 1-4, in Fig. 3(b), $\left(\epsilon_{\mu}\right)=(1,1,-1,-1)$ for the experimentally found magnetic structure in the AFI. The local field $\left(H_{\mu}\right)$ $=\left(H^{+}, H^{+}, H^{-}, H^{-}\right)$with $H^{ \pm}=H_{0} \pm H_{e}$, where $H_{0}$ is the anisotropic field in the material and $H_{e}$ is an external field applied along the up spin direction. Limiting the range of exchange interactions to the fourth nearest neighbors, there are seven distinct exchange constants: $J_{\alpha}, J_{\beta}, J_{\gamma}, J_{\delta}, J_{\epsilon}, J_{\zeta}$, and $J_{\eta}$ [refer to Fig. 3(b)]. Their contributions to various spin pairs are tabulated in Table II. Now the matrix $\mathrm{J}(\boldsymbol{\kappa})$ can be readily calculated by collecting contributions to spin pairs $(\mu \nu)$ according to Eq. (A3). Notice that spin pairs $(\mu \mu)$ are identically connected by $J_{\eta}$, therefore $J_{11}=J_{22}=J_{33}=J_{44}$. Spin pairs (23), (24), and (34) are identically connected as spin pairs (14), (31), and (21), respectively. This leads to $\mathrm{J}_{23}=J_{14}, J_{24}=J_{31}$, and $J_{34}=J_{21}$. Recall also that $J(\boldsymbol{\kappa})$ is Hermitian, and the matrix has only four independent elements:

$$
\mathrm{J}(\boldsymbol{\kappa})=\left(\begin{array}{llll}
A & B & C & D \\
B^{*} & A & D & C^{*} \\
C^{*} & D^{*} & A & B^{*} \\
D^{*} & C & B & A
\end{array}\right) .
$$

They are given by

$$
\begin{gathered}
A=\mathrm{J}_{11}(\boldsymbol{\kappa})=2 J_{\eta} \cos (2 \pi h) \\
B=J_{12}(\boldsymbol{\kappa})=J_{\alpha} \exp [i 2 \pi \phi(h-k)] \\
+J_{\gamma} \exp [i 2 \pi(-\psi h-\phi k)] \\
+J_{\epsilon} \exp [i 2 \pi(\phi h+\psi k)] \\
C=J_{13}(\boldsymbol{\kappa})=2 J_{\beta} \cos (\pi l) \exp [i 2 \pi(\phi h+\theta k)] \\
+2 J_{\delta} \cos (\pi l) \exp [i 2 \pi(-\psi h+\theta k)] \\
D=J_{14}(\boldsymbol{\kappa})=4 J_{\zeta} \cos (\pi l) \cos (\pi k),
\end{gathered}
$$

\begin{tabular}{|c|c|c|c|}
\hline$J_{j \nu, 0 \mu}$ & $\mu$ & $\nu$ & $\mathbf{R}_{j \nu}-\mathbf{R}_{0 \mu}$ \\
\hline \multirow[t]{2}{*}{$J_{\alpha}$} & 1 & 2 & $\mathbf{x}^{\mathrm{a}}$ \\
\hline & 3 & 4 & $-\mathbf{x}$ \\
\hline \multirow[t]{4}{*}{$J_{\beta}$} & 1 & 3 & $\mathbf{y}^{b}-\mathbf{c}_{m} / 2$ \\
\hline & 1 & 3 & $\mathbf{y}+\mathbf{c}_{m} / 2$ \\
\hline & 2 & 4 & $-\mathbf{y}-\mathbf{c}_{m} / 2$ \\
\hline & 2 & 4 & $-\mathbf{y}+\mathbf{c}_{m} / 2$ \\
\hline$J_{\gamma}$ & 1 & 2 & $\mathbf{x}-\mathbf{a}_{m}$ \\
\hline \multirow[t]{4}{*}{$J_{\delta}$} & 1 & 3 & $\mathbf{y}-\mathbf{a}_{m}-\mathbf{c}_{m} / 2$ \\
\hline & 1 & 3 & $\mathbf{y}-\mathbf{a}_{m}+\mathbf{c}_{m} / 2$ \\
\hline & 2 & 4 & $\mathbf{a}_{m}-\mathbf{y}-\mathbf{c}_{m} / 2$ \\
\hline & 2 & 4 & $\mathbf{a}_{m}-\mathbf{y}+\mathbf{c}_{m} / 2$ \\
\hline$J_{\epsilon}$ & 1 & 2 & $\mathbf{x}+\mathbf{b}_{m}$ \\
\hline \multirow[t]{8}{*}{$J_{\zeta}$} & 1 & 4 & $\mathbf{b}_{m} / 2-\mathbf{c}_{m} / 2$ \\
\hline & 1 & 4 & $\mathbf{b}_{m} / 2+\mathbf{c}_{m} / 2$ \\
\hline & 1 & 4 & $-\mathbf{b}_{m} / 2-\mathbf{c}_{m} / 2$ \\
\hline & 1 & 4 & $-\mathbf{b}_{m} / 2+\mathbf{c}_{m} / 2$ \\
\hline & 2 & 3 & $\mathbf{b}_{m} / 2-\mathbf{c}_{m} / 2$ \\
\hline & 2 & 3 & $\mathbf{b}_{m} / 2+\mathbf{c}_{m} / 2$ \\
\hline & 2 & 3 & $-\mathbf{b}_{m} / 2-\mathbf{c}_{m} / 2$ \\
\hline & 2 & 3 & $-\mathbf{b}_{m} / 2+\mathbf{c}_{m} / 2$ \\
\hline \multirow[t]{2}{*}{$J_{\eta}$} & 1 & 1 & $\mathbf{a}_{m}$ \\
\hline & 1 & $1^{\mathrm{c}}$ & $-\mathbf{a}_{m}$ \\
\hline
\end{tabular}

where $\boldsymbol{\kappa}=(h k l)$ is indexed in the monoclinic reciprocal lattice, $\phi=2 \delta+1 / 3, \psi=2 / 3-2 \delta, \theta=1 / 6-2 \delta$, and $\delta=0.026$. Notice that only $B$ and $C$ are complex. $A$ and $D$ are real.

The diagonal terms of $L$ in the first line of Eq. (A4) are

$$
\delta_{\mu \nu}\left[H_{\mu}+2 S\left(J_{\alpha}+J_{\gamma}+J_{\epsilon}+2 J_{\eta}-2 J_{\beta}-2 J_{\delta}-4 J_{\zeta}\right)\right] .
$$

TABLE II. Spin pairs connected by the seven exchange constants.

${ }^{\mathrm{a}} \mathbf{x} \equiv \mathbf{R}_{02}-\mathbf{R}_{01}=(1 / 3+2 \delta)\left(\mathbf{a}_{m}-\mathbf{b}_{m}\right), \quad$ where $\delta=0.026$. For $(\mu \nu)=(21), \mathbf{R}_{j \nu}-\mathbf{R}_{0 \mu}=-\mathbf{x}$. Exchanging $\mu$ and $\nu$ switches the sign for $\mathbf{R}_{j \nu}-\mathbf{R}_{0 \mu}$.

$\mathrm{b}_{\mathbf{y}} \equiv(1 / 3+2 \delta) \mathbf{a}_{m}+(1 / 6-2 \delta) \mathbf{b}_{m}$.

${ }^{\mathrm{c}} J_{\eta}$ has identical contributions for $(\mu \nu)=(22)$, (33), and (44) spin pairs.

When there is no external field, $H_{\mu}=H_{0}$ no longer depends on $\mu$. In this case,

$$
\mathrm{L}(\boldsymbol{\kappa})=2 S\left(\begin{array}{cccc}
F & -B & C & D \\
-B^{*} & F & D & C^{*} \\
C^{*} & D & F & -B^{*} \\
D & C & -B & F
\end{array}\right) \text {, }
$$

where

$$
F=J_{\alpha}+J_{\gamma}+J_{\epsilon}+2 J_{\eta}-2 J_{\beta}-2 J_{\delta}-4 J_{\zeta}+H_{0} / 2 S-A .
$$

The eigenvalue equation for $E L$ is

$$
\left|\begin{array}{cccc}
F-\lambda^{\prime} & -B & C & D \\
-B^{*} & F-\lambda^{\prime} & D & C^{*} \\
C^{*} & D & F+\lambda^{\prime} & -B^{*} \\
D & C & -B & F+\lambda^{\prime}
\end{array}\right|=0,
$$

where $\lambda^{\prime} \equiv \lambda / 2 S$. It can be verified that the linear and cubic terms in $\lambda^{\prime}$ vanish and therefore the eigenvalue equation is quadratic in $\lambda^{\prime 2}$ :

$$
\lambda^{\prime 4}-2 b(\boldsymbol{\kappa}) \lambda^{\prime 2}+|\mathrm{L}(\boldsymbol{\kappa})|=0,
$$


where both the determinant $|\mathrm{L}(\boldsymbol{\kappa})|$ and

$$
b(\boldsymbol{\kappa}) \equiv F^{2}+|B|^{2}-|C|^{2}-D^{2}
$$

are real functions of $\boldsymbol{\kappa}$. The consequence of this is that in the absence of an external magnetic field, there are two branches of doubly degenerate spin wave modes with a dispersion relation

$$
\hbar \omega(\boldsymbol{\kappa})=2 S[b(\boldsymbol{\kappa}) \pm c(\boldsymbol{\kappa})]^{1 / 2},
$$

where

$$
\begin{aligned}
c^{2}(\boldsymbol{\kappa}) \equiv & b^{2}(\boldsymbol{\kappa})-|\mathrm{L}(\boldsymbol{\kappa})| \\
= & 4 F^{2}|B|^{2}+4|C|^{2} D^{2}-2|B|^{2}|C|^{2} \\
& +B^{2} C^{* 2}+B^{* 2} C^{2}+4 F D\left(B C^{*}+B^{*} C\right) .
\end{aligned}
$$

*Current address: MST-10, Los Alamos National Laboratory, Los Alamos, NM 87545.

${ }^{1}$ N. F. Mott, Metal-Insulator Transitions, 2nd ed. (Taylor \& Francis Ltd., London, 1990).

${ }^{2}$ J. Kanamori, Prog. Theor. Phys. 30, 275 (1963); M. C. Gutzwiller, Phys. Rev. Lett. 10, 159 (1963); Phys. Rev. 134, A923 (1964); J. Hubbard, Proc. R. Soc. London, Ser. A 276, 238 (1963).

${ }^{3}$ J. Hubbard, Proc. R. Soc. London, Ser. A 281, 401 (1964).

${ }^{4}$ D. M. Edwards and A. C. Hewson, Rev. Mod. Phys. 40, 810 (1968).

${ }^{5}$ M. Cyrot, Physica B\&C 91, 141 (1977).

${ }^{6}$ W. F. Brinkman and T. M. Rice, Phys. Rev. B 2, 4302 (1970).

${ }^{7} \mathrm{P}$. W. Anderson, in Frontiers and Borderlines in Many Particle Physics (North-Holland, Amsterdam, 1987).

${ }^{8}$ J. Zaanen, G. A. Sawatzky, and J. W. Allen, Phys. Rev. Lett. 55, 418 (1985).

${ }^{9}$ See, e.g., A. Fujimori, J. Phys. Chem. Solids 53, 1595 (1992); A. Fujimori, I. Hase, Y. Tokura, M. Abbate, F. M. de Groot, J. C. Fuggle, H. Eisaki, and S. Uchida, Physica B 186-188, 191 (1993).

${ }^{10}$ W. Metzner and D. Vollhardt, Phys. Rev. Lett. 62, 324 (1989).

${ }^{11}$ M. Jarrel, Phys. Rev. Lett. 69, 168 (1992); M. J. Rozenberg, X. Y. Zhang, and G. Kotliar, ibid. 69, 1236 (1992); A. Georges and W. Krauth, ibid. 69, 1240 (1992).

${ }^{12}$ M. J. Rozenberg, G. Kotliar, and X. Y. Zhang, Phys. Rev. B 49, 10181 (1994); X. Y. Zhang, M. J. Rozenberg, and G. Kotliar, Phys. Rev. Lett. 70, 1666 (1993).

${ }^{13}$ T. Moriya, Spin Fluctuations in Itinerant Electron Magnetism (Springer-Verlag, Berlin, 1985).

${ }^{14}$ K. K. Murata and S. Doniach, Phys. Rev. Lett. 29, 285 (1972).

${ }^{15}$ T. Moriya and A. Kawabata, J. Phys. Soc. Jpn. 34, 639 (1973); 35, 669 (1973).

${ }^{16}$ T. V. Ramakrishnan, Phys. Rev. B 10, 4014 (1974).

${ }^{17}$ G. G. Lonzarich, J. Magn. Magn. Mater. 54-57, 612 (1986).

${ }^{18}$ H. Hasegawa and T. Moriya, J. Phys. Soc. Jpn. 36, 1542 (1974).

${ }^{19}$ D. B. McWhan, A. Menth, J. P. Remeika, W. F. Brinkman, and T. M. Rice, Phys. Rev. B 7, 1920 (1973).

${ }^{20}$ J. Feinleib and W. Paul, Phys. Rev. 155, 841 (1967).

${ }^{21}$ H. Kuwamoto, J. M. Honig, and J. Appel, Phys. Rev. B 22, 2626 (1980).

${ }^{22}$ D. B. McWhan and J. P. Remeika, Phys. Rev. B 2, 3734 (1970).

${ }^{23}$ P. D. Dernier and M. Marezio, Phys. Rev. B 2, 3771 (1970).

${ }^{24}$ R. M. Moon, Phys. Rev. Lett. 25, 527 (1970).

${ }^{25}$ I. Nebenzahl and M. Weger, Phys. Rev. 184, 936 (1969).

${ }^{26}$ L. F. Mattheiss, J. Phys.: Condens. Matter 6, 6477 (1994).

${ }^{27}$ J. C. Slater, Phys. Rev. 82, 538 (1951).

${ }^{28}$ M. Cyrot and P. Lacour-Gayet, Solid State Commun. 11, 1767 (1972).

${ }^{29}$ T. Moriya and H. Hasegawa, J. Phys. Soc. Jpn. 48, 1490 (1980).
${ }^{30}$ J. Spalek, A. Datta, and J. M. Honig, Phys. Rev. Lett. 59, 728 (1987).

${ }^{31}$ R. E. Word, S. A. Werner, W. B. Yelon, J. M. Honig, and S. Shivashankar, Phys. Rev. B 23, 3533 (1981).

${ }^{32}$ M. Yethiraj, S. A. Werner, W. B. Yelon, and J. M. Honig, Phys. Rev. B 36, 8675 (1987).

${ }^{33}$ W. Bao, C. Broholm, S. A. Carter, T. F. Rosenbaum, G. Aeppli, S. F. Trevino, P. Metcalf, J. M. Honig, and J. Spalek, Phys. Rev. Lett. 71, 766 (1993).

${ }^{34}$ G. Aeppli, W. Bao, C. Broholm, S-W. Cheong, P. Dai, S. M. Hayden, T. E. Mason, H. A. Mook, T. G. Perring, and J. diTusa, in Spectroscopy of Mott Insulators and Correlated Metals, edited by A. Fujimori and Y. Tokura (Springer, Berlin, 1995).

${ }^{35}$ C. Broholm, G. Aeppli, S.-H. Lee, W. Bao, and J. F. Ditusa, J. Appl. Phys. 79, 5023 (1996).

${ }^{36}$ W. Bao, C. Broholm, J. M. Honig, P. Metcalf, and S. F. Trevino, Phys. Rev. B 54, R3726 (1996).

${ }^{37}$ W. Bao, C. Broholm, G. Aeppli, P. Dai, J. M. Honig, and P. Metcalf, Phys. Rev. Lett. 78, 507 (1997).

${ }^{38}$ W. Bao, C. Broholm, G. Aeppli, S. A. Carter, P. Dai, C. D. Frost, J. M. Honig, and P. Metcalf, J. Magn. Magn. Mater. 177181, 283 (1998).

${ }^{39}$ W. Bao, Ph.D. dissertation, The Johns Hopkins University, 1995 (unpublished).

${ }^{40}$ J. B. Goodenough, Prog. Solid State Chem. 5, 145 (1972).

${ }^{41}$ W. R. Robinson, Acta Crystallogr., Sect. B: Struct. Crystallogr. Cryst. Chem. B31, 1153 (1975).

${ }^{42}$ S. Chen, J. E. Hahn, C. E. Rice, and W. R. Robinson, J. Solid State Chem. 44, 192 (1982).

${ }^{43}$ J. E. Keem et al., Am. Ceram. Soc. Bull. 56, 1022 (1977); H. R. Harrison, R. Aragon, and C. J. Sandberg, Mater. Res. Bull. 15, 571 (1980).

${ }^{44}$ S. A. Shivashankar et al., J. Electrochem. Soc. 128, 2472 (1981); 129, 1641(E) (1982).

${ }^{45}$ S. A. Shivashankar and J. M. Honig, Phys. Rev. B 28, 5695 (1983).

${ }^{46}$ S. A. Carter, T. F. Rosenbaum, P. Metcalf, J. M. Honig, and J. Spalek, Phys. Rev. B 48, 16841 (1993).

${ }^{47}$ S. A. Carter, T. F. Rosenbaum, J. M. Honig, and J. Spalek, Phys. Rev. Lett. 67, 3440 (1991).

${ }^{48}$ S. A. Carter, J. Yang, T. F. Rosenbaum, J. Spalek and J. M. Honig, Phys. Rev. B 43, 607 (1991).

${ }^{49}$ S. A. Carter, T. F. Rosenbaum, M. Lu, H. M. Jaeger, P. Metcalf, J. M. Honig, and J. Spalek, Phys. Rev. B 49, 7898 (1994).

${ }^{50}$ W. Bao, C. Broholm, and S. F. Trevino, Rev. Sci. Instrum. 66, 1260 (1995).

${ }^{51}$ D. C. Tennant, Rev. Sci. Instrum. 59, 380 (1988).

${ }^{52}$ S. M. Shapiro and N. J. Chesser, Nucl. Instrum. Methods 101, 183 (1972). 
${ }^{53}$ Similar "contamination" from main neutron beam occurs in, e.g., the $18 \mathrm{meV}$ scans in Fig. 1 of Ref. 36.

${ }^{54}$ S. W. Lovesey, Theory of Neutron Scattering from Condensed Matter (Clarendon Press, Oxford, 1984).

${ }^{55}$ R. E. Watson and A. J. Freeman, Acta Crystallogr. 14, 27 (1961)

${ }^{56}$ N. J. Chesser and J. D. Axe, Acta Crystallogr., Sect. A: Cryst. Phys., Diffr., Theor. Gen. Crystallogr. A29, 160 (1973).

${ }^{57}$ Y. Ishikawa, Y. Noda, Y. J. Uemura, C. F. Majkrzak, and G. Shirane, Phys. Rev. B 31, 5884 (1985).

${ }^{58}$ M. J. Cooper and R. Nathans, Acta Crystallogr. 23, 357 (1967); Acta Crystallogr., Sect. A: Cryst. Phys., Diffr., Theor. Gen. Crystallogr. A24, 481 (1968); A24, 619 (1968); M. J. Cooper, ibid. A24, 624 (1968)

${ }^{59}$ S. A. Werner and R. Pynn, J. Appl. Phys. 42, 4736 (1971).

${ }^{60}$ D. B. McWhan and T. M. Rice, Phys. Rev. Lett. 22, 887 (1969).

${ }^{61}$ A. C. Gossard, D. B. McWhan, and J. P. Remeika, Phys. Rev. B 2, 3762 (1970).

${ }^{62}$ M. Nakahira, S. Horiuchi, and H. Ooshima, J. Appl. Phys. 41, 836 (1970).

${ }^{63}$ Y. Ueda, K. Kosuge, S. Kachi, T. Shinjo, and T. Takada, Mater. Res. Bull. 12, 87 (1977).

${ }^{64}$ J. Dumas and C. Schlenker, J. Magn. Magn. Mater. 7, 252 (1978).

${ }^{65}$ Y. Ueda, K. Kosuge, S. Kachi, and T. Takada, J. Phys. (Paris), Colloq. 40, C2-275 (1979).

${ }^{66}$ Y. Ueda, K. Kosuge, and S. Kachi, J. Solid State Chem. 31, 171 (1980)

${ }^{67}$ W. M. Lomer, Proc. Phys. Soc. London 80, 489 (1962).

${ }^{68}$ A. W. Overhauser, Phys. Rev. 128, 1437 (1962).

${ }^{69}$ P. A. Fedders and P. C. Martin, Phys. Rev. 143, 245 (1966).

${ }^{70}$ T. Wolenski, M. Grodzicki, and J. Appel, Phys. Rev. B 58, 303 (1998); Q. Si (private communication).

${ }^{71}$ D. B. McWhan and T. M. Rice, Phys. Rev. Lett. 19, 846 (1967).

${ }^{72}$ Y. Ueda, K. Kosuge, S. Kachi, H. Yasuoka, H. Nishihara, and A. Heidemann, J. Phys. Chem. Solids 39, 1281 (1978).

${ }^{73}$ R. M. Moon, T. Riste, and W. C. Koehler, Phys. Rev. 181, 920 (1969).

${ }^{74}$ P. W. Anderson, Phys. Rev. B 30, 1549 (1984).

${ }^{75}$ A. Husmann, T. F. Rosenbaum, and J. M. Honig (unpublished).

${ }^{76}$ S. H. Liu, Phys. Rev. B 2, 2664 (1970).

${ }^{77}$ J. B. Sokoloff, Phys. Rev. 185, 770 (1969); 185, 783 (1969).

${ }^{78}$ L. M. Corliss, J. M. Hastings, and R. Weiss, Phys. Rev. Lett. 3, 211 (1959).

${ }^{79}$ E. Fawcett, Rev. Mod. Phys. 60, 209 (1988).

${ }^{80}$ S. K. Sinha, S. H. Liu, L. D. Muhlestein, and N. Wakabayashi, Phys. Rev. Lett. 23, 311 (1969).

${ }^{81}$ J. Als-Nielsen, J. D. Axe, and G. Shirane, J. Appl. Phys. 42, 1666 (1971).

${ }^{82}$ E. D. Jones, Phys. Rev. 137, A978 (1965).

${ }^{83}$ A. Menth and J. P. Remeika, Phys. Rev. B 2, 3756 (1970).

${ }^{84}$ J. A. Hertz, Phys. Rev. B 14, 1165 (1976).

${ }^{85}$ S. Chakravarty, B. I. Halperin, and D. R. Nelson, Phys. Rev. Lett. 60, 1057 (1988).

${ }^{86}$ S. Sachdev and J. Ye, Phys. Rev. Lett. 69, 2411 (1992).

${ }^{87}$ A. J. Millis, Phys. Rev. B 48, 7183 (1993).

${ }^{88}$ Constant energy scans at $1.8,3,6,9,12,15$, and $18 \mathrm{meV}$ were used for the $1.4 \mathrm{~K}$ fitting; $1.4,2.5,4,9$, and $15 \mathrm{meV}$ for $11 \mathrm{~K}$; $1.5,2.5,4,9$, and 15 for $30 \mathrm{~K} ; 1.5,7$, and $12 \mathrm{meV}$ for $50 \mathrm{~K} ; 4$, $7,10,13$, and $17 \mathrm{meV}$ for $70 \mathrm{~K} ; 5,10,15,20$, and $28 \mathrm{meV}$ for $90 \mathrm{~K} ; 5,15,24$, and $28 \mathrm{meV}$ for $110 \mathrm{~K} ; 1.5,7$, and $12 \mathrm{meV}$ for
$130 \mathrm{~K} ; 5,10,15$, and 28 for $150 \mathrm{~K}$; and $1.8,3,6,9,12$, and 18 meV for $200 \mathrm{~K}$.

${ }^{89}$ M. E. Fisher, Rev. Mod. Phys. 46, 597 (1974).

${ }^{90}$ B. Halperin and P. C. Hohenberg, Phys. Rev. Lett. 19, 700 (1967); Phys. Rev. 177, 952 (1969).

${ }^{91}$ S. M. Hayden et al., Phys. Rev. Lett. 66, 821 (1991); see also, B. Keimer et al., ibid. 67, 1930 (1991).

${ }^{92}$ A. Schroder, G. Aeppli, E. Bucher, R. Ramazashvili, and P. Coleman, Phys. Rev. Lett. 80, 5623 (1998); M. C. Aronson et al., ibid. 75, 725 (1995).

${ }^{93}$ C. M. Varma, P. B. Littlewood, and S. Schmitt-Rink, Phys. Rev. Lett. 63, 1996 (1989).

${ }^{94}$ These constants are related to parameters $\Gamma$ and $\bar{A}$ which we used in Ref. 36 by the relation $\Gamma=C a_{2}^{z} k_{B}$ and $\bar{A}=a_{2}^{z} / a_{1}$.

${ }^{95}$ K. Nakayama and T. Moriya, J. Phys. Soc. Jpn. 56, 2918 (1987).

${ }^{96}$ H. Hasegawa, J. Phys. Soc. Jpn. 38, 107 (1975); T. Moriya and K. Ueda, ibid. 63, 1871 (1994).

${ }^{97}$ H.-Y. Lau, L. M. Corliss, A. Delapalme, J. M. Hastings, R. Nathana, and A. Tucciarone, J. Appl. Phys. 41, 1384 (1970).

${ }^{98}$ H. Sato and K. Maki, Int. J. Magn. 6, 193 (1974).

${ }^{99}$ D. R. Noakes, T. M. Holden, E. Fawcett, and P. C. de Camargo, Phys. Rev. Lett. 65, 369 (1990).

${ }^{100}$ T. E. Mason, G. Aeppli, and H. A. Mook, Phys. Rev. Lett. 68, 1414 (1992); G. Aeppli et al., Science 278, 1432 (1997).

${ }^{101}$ A. J. Millis, H. Monien, and D. Pines, Phys. Rev. B 42, 167 (1990).

${ }^{102}$ N. Bulut, D. Hone, D. J. Scalapino, and N. E. Bickers, Phys. Rev. B 41, 1797 (1990).

${ }^{103}$ H. Monien, D. Pines, and M. Takigawa, Phys. Rev. B 43, 258 (1991).

${ }^{104}$ G. G. Lonzarich, J. Magn. Magn. Mater. 45, 43 (1984).

${ }^{105}$ Y. Takahashi and T. Moriya, J. Phys. Soc. Jpn. 54, 1592 (1985).

${ }^{106}$ Y. Ishikawa, Y. Noda, C. Fincher, and G. Shirane, Phys. Rev. B 25, 254 (1982).

${ }^{107}$ E. J. Samuelsen, M. T. Hutchings, and G. Shirane, Physica (Amsterdam) 48, 13 (1970).

${ }^{108}$ E. J. Samuelsen and G. Shirane, Phys. Status Solidi 42, 241 (1970).

${ }^{109}$ B. N. Brockhouse, J. Chem. Phys. 21, 961 (1953); L. M. Corliss, J. M. Hastings, R. Nathans, and G. Shirane, J. Appl. Phys. 36, 1099 (1965).

${ }^{110}$ C. G. Shull, W. A. Straser, and E. O. Wollan, Phys. Rev. 83, 333 (1951); R. Nathans, S. J. Pickart, H. A. Alperin, and P. J. Brown, Phys. Rev. 136, A1641 (1964).

${ }^{111}$ E. J. Samuelsen, Physica (Amsterdam) 43, 353 (1969).

${ }^{112}$ E. P. Warekois, J. Appl. Phys. 31, 346 (1960).

${ }^{113}$ A. W. Sáenz, Phys. Rev. 125, 1940 (1962).

${ }^{114}$ The values of individual exchange constants depend strongly on how many of the seven exchanges are included in theoretical fits to our data and they are correlated. Thus, our data, as well as those by Word et al., ${ }^{31}$ cannot determine them individually. The sum $J_{\beta}+J_{\delta}+2 J_{\zeta}=45.9-2 \times 4.76 \approx 35$ from Ref. 31 , close to our value. The definition of the anisotropy $A_{\mu}$ of Ref. 31 was not given.

${ }^{115}$ E. F. Bertaut, C. R. Acad. Sci. Paris 252, 252 (1961); 252, 2032 (1961).

${ }^{116}$ N. Menyuk and K. Dwight, J. Phys. Chem. Solids 25, 1031 (1964).

${ }^{117}$ T. M. Rice, in Spectroscopy of Mott Insulators and Correlated Metals, edited by A. Fujimori and Y. Tokura (Springer, Berlin, 1995). 
${ }^{118}$ M. Takigawa, E. T. Ahrens, and Y. Ueda, Phys. Rev. Lett. 76, 283 (1996).

${ }^{119}$ C. Castellani, C. R. Natoli, and J. Ranninger, Phys. Rev. B 18, 4945 (1978); 18, 4967 (1978); 18, 5001 (1978).

${ }^{120}$ S. K. Ma, Modern Theory of Critical Phenomena (Benjamin, Reading, 1976).

${ }^{121}$ M. Cyrot, J. Phys. (Paris) 33, 125 (1972); Q. Si, Y. Zha, and K. Levin, J. Appl. Phys. 76, 6935 (1994).

${ }^{122}$ B. I. Shraiman and E. D. Siggia, Phys. Rev. Lett. 62, 1564 (1989); H. J. Schulz, ibid. 64, 1445 (1990); S. Sarker, C. Jayaprakash, H. R. Krishnamurthy, and W. Wenzel, Phys. Rev. B 43, 8775 (1991).

${ }^{123}$ P. W. Anderson, Adv. Phys. 46, 1 (1997).

${ }^{124}$ N. F. Mott, Rev. Mod. Phys. 40, 677 (1968).

${ }^{125}$ J. H. de Boer and E. J. Verwey, Proc. R. Soc. London 49, 59 (1937).

${ }^{126}$ D. B. McWhan, J. P. Remeika, S. D. Bader, B. B. Triplett, and
N. E. Phillips, Phys. Rev. B 7, 3079 (1973).

${ }^{127}$ D. B. McWhan, T. M. Rice, and J. P. Remeika, Phys. Rev. Lett. 23, 1384 (1969).

${ }^{128}$ A. Jayaraman, D. B. McWhan, J. P. Remeika, and P. D. Dernier, Phys. Rev. B 2, 3751 (1970).

${ }^{129}$ P. W. Anderson, Solid State Phys. 14, 99 (1963).

${ }^{130}$ K. I. Kugel and D. I. Khomskii, Sov. Phys. JETP 37, 725 (1973).

${ }^{131}$ M. Cyrot and C. Lyon-Caen, J. Phys. C 6, L247 (1973).

${ }^{132}$ M. Cyrot and C. Lyon-Caen, J. Phys. (Paris), Colloq. 4, 183 (1976).

${ }^{133}$ H. F. Pen, J. van den Brink, D. I. Khomskii, and G. A. Sawatzky, Phys. Rev. Lett. 78, 1323 (1997).

${ }^{134}$ L. F. Feiner, A. M. Oles, and J. Zaanen, Phys. Rev. Lett. 78, 2799 (1997).

${ }^{135}$ W. Bao, J. D. Axe, C. H. Chen, and S.-W. Cheong, Phys. Rev. Lett. 78, 543 (1997).

${ }^{136}$ T. Holstein and H. Primakoff, Phys. Rev. 58, 1098 (1940). 